\title{
Multispacecraft observations of the terrestrial bow shock and magnetopause during extreme solar wind disturbances
}

\author{
M. Tátrallyay ${ }^{1}$, G. Erdôs ${ }^{1}$, Z. Németh ${ }^{1}$, M. I. Verigin ${ }^{2}$, and S. Vennerstrom ${ }^{3}$ \\ ${ }^{1}$ Wigner RCP, RMKI (formerly KFKI Res. Inst. Particle and Nuclear Physics), 1525 Budapest, P.O.B. 49, Hungary \\ ${ }^{2}$ Space Research Institute RAS, Moscow, Russia \\ ${ }^{3}$ National Space Institute, TU Denmark, Kgs. Lyngby, Denmark \\ Correspondence to: M. Tátrallyay (tatrallyay.mariella@wigner.mta.hu)
}

Received: 26 June 2011 - Revised: 8 June 2012 - Accepted: 22 October 2012 - Published: 12 December 2012

\begin{abstract}
Three events are discussed from the declining phase of the last solar cycle when the magnetopause and/or the bow shock were observed unusually close to the Earth due to major interplanetary disturbances. The observed extreme locations of the discontinuities are compared with the predictions of three magnetopause and four bow shock models which describe them in considerably different ways using statistical methods based on observations. A new 2-D magnetopause model is introduced (based on Verigin et al., 2009) which takes into account the pressure of the compressed magnetosheath field raised by the interplanetary magnetic field (IMF) component transverse to the solar wind flow. The observed magnetopause crossings could be predicted with a reasonable accuracy $\left(0.1-0.2 R_{\mathrm{E}}\right)$ by one of the presented models at least. For geosynchronous magnetopause crossings observed by the GOES satellites, (1) the new model provided the best predictions when the IMF was extremely large having a large negative $B_{\mathrm{Z}}$ component, and (2) the predictions of the model of Shue et al. (1998) agreed best with the observations when the solar wind dynamic pressure was extremely large. The magnetopause crossings close to the cusp observed by the Cluster spacecraft were best predicted by the 3-D model of Lin et al. (2010). The applied empirical bow shock models and the 3-D semi-empiric bow shock model combined with magnetohydrodynamic (MHD) solution proved to be insufficient for predicting the observed unusual bow shock locations during large interplanetary disturbances. The results of a global 3-D MHD model were in good agreement with the Cluster observations on 17 January 2005, but they did not predict the bow shock crossings on 31 October 2003.
\end{abstract}

Keywords. Magnetospheric physics (Magnetopause, cusp, and boundary layers)

\section{Introduction}

The terrestrial magnetopause is the result of the interaction between the supersonic solar wind and the Earth's magnetic field. The location of the magnetopause is one of the most important parameters in space physics because it is the boundary that separates the magnetospheric plasma from the solar wind and determines the size of the magnetosphere. As the velocity of the solar wind exceeds the velocity of sonic, Alfvénic, and magnetosonic waves in interplanetary space, a bow shock forms in front of the magnetopause where plasma parameters suddenly change; velocity decreases while density, temperature, and the tangential component of the magnetic field increase. The region between the bow shock and the magnetopause is the magnetosheath.

Since Ferraro (1952) first calculated the size of the magnetosphere, space physicists have given much effort to modelling the location and the shape of the magnetopause under different solar wind conditions. Most of the early studies supposed that the location of the magnetopause depends solely on solar wind dynamic pressure $P_{\mathrm{d}}$. Fairfield (1971) recognized that the IMF (interplanetary magnetic field) orientation can also affect the magnetopause location. Later, several twodimensional empirical models were developed on the basis of mainly low-latitude magnetopause crossings assuming rotational symmetry. Several two-dimensional models (cf. Howe and Binsack, 1972; Petrinec et al., 1991; Sibeck et al., 1991; Petrinec and Russell, 1993; Shue et al., 1997; Verigin et al., 
2009) define the magnetopause size and shape in limited parameter ranges, and they are not valid for extreme solar wind conditions, which are rarely observed. Other models include extreme values of interplanetary parameters $\left(P_{\mathrm{d}}\right.$ and IMF $B_{\mathrm{Z}}$ ), and they try to predict unusual magnetopause locations beyond average conditions (cf. Roelof and Sibeck, 1993; Petrinec and Russell, 1996; Shue et al., 1998; Chao et al., 2002). Several studies compared the forecasting capabilities of different models for magnetopause crossings under extreme solar wind conditions (cf. Shue et al., 1998; Yang et al., 2002; Suvorova et al., 2005).

Shue et al. (1998) improved their earlier model (Shue et al., 1997), when comparing it with the model of Petrinec and Russell (1996), in order to get better predictions for magnetopause crossings observed by Geotail and Interball 1 along the flank and by geosynchronous satellites on the dayside under extreme solar wind conditions. Yang et al. (2002) compared the models of Petrinec and Russell (1996), Shue et al. (1998), and Chao et al. (2002) when investigating magnetopause crossings on geosynchronous orbit on several days. A more extended comparison of these three models was made by Suvorova et al. (2005), including two more models (Kuznetsov and Suvorova, 1998; Dmitriev and Suvorova, 2000), when investigating the necessary solar wind conditions for geosynchronous magnetopause crossings. They found that the magnetopause location is not influenced by IMF $B_{\mathrm{Z}}$ when $B_{\mathrm{Z}}$ has large positive value and $P_{\mathrm{d}}>21 \mathrm{nPa}$, or when $B_{\mathrm{Z}}$ has large negative value and $P_{\mathrm{d}}<4.8 \mathrm{nPa}$. Suvorova et al. (2005) explained the first case with pressure balance and the second case with the saturation of the $B_{\mathrm{Z}}$ influence.

As more satellites sampled the Earth at different orbits reaching higher latitudes, parameters raising asymmetry (deviations from the cylindrical model) were also taken into account. Formisano et al. (1979) determined the average size and shape of the magnetopause in Solar Magnetic (SM) coordinates for three different values of the tilt angle $\phi$ of the geomagnetic dipole. They used a limited number of magnetopause crossings in the high-latitude region, which were normalized for solar wind dynamic pressure $P_{\mathrm{d}}$. Boardsen et al. (2000) developed a quantitative empirical high-latitude model parameterized by $P_{\mathrm{d}}$, IMF $B_{\mathrm{z}}$, and $\phi$ in Geocentric Solar Magnetospheric (GSM) coordinates, which was only valid in limited regions. Lin et al. (2010) presented a global three-dimensional asymmetric magnetopause model taking into account the effect of the tilt angle in addition to the solar wind parameters. Based on an extended dataset, they found that their new model provided the smallest standard deviation for the magnetopause location when compared with eight other models.

The first bow shock models also assumed cylindrical symmetry relative to the solar wind direction. After the gasdynamic simulation of Spreiter et al. (1966), several twodimensional empirical models were developed for the average position and shape of the bow shock by fitting the equa- tion of a general conic of revolution with different sets of observations (cf. Fairfield, 1971; Formisano et al., 1971; Slavin and Holzer, 1981; Farris et al., 1991; Cairns et al., 1995). When ordering the observations according to solar wind dynamic pressure and/or Mach number, it became obvious that the standoff distance and the flaring angle of the tail are influenced by these parameters.

Formisano (1979) derived a three-dimensional empirical bow shock model including a large number of high-latitude bow shock crossings which were normalized to average solar wind dynamic pressure. This model was modified by Nemecek and Safrankova (1991) and later by Jerab et al. (2005) by using different forms for the effect of the Mach numbers. Peredo et al. (1995) presented a three-dimensional empirical model explicitly parameterized by the Alfvénic Mach number. The observed bow shock crossings were pressure normalized and rotated into Geocentric InterPlanetary Medium coordinates (GIPM defined by Bieber and Stone, 1979) where the $X$-axis points opposite to the solar wind direction and the IMF is in the X-Y plane. In the GIPM frame of reference, Verigin et al. (2001a,b, 2003) developed a threedimensional semi-empiric bow shock model combined with MHD (magnetohydrodynamic) solution.

Merka et al. (2003) compared the predictions of different bow shock models with observations collected by the IMP 8 spacecraft over 12 years. The accuracy of the models was estimated by the ratio of the predicted to observed radial distances. They found that the model of Formisano et al. (1979) provided the most accurate bow shock location when all data were used. For typical solar wind conditions, the best estimations were provided by the paraboloid surface given by Cairns et al. (1995) when applying the standoff distance predicted either by Farris and Russell (1994) or by Cairns and Lyon (1995). The predictions of the model by Peredo et al. (1995) were about $20 \%$ larger, while the predictions of the model by Nemecek and Safrankova (1991) were more than $10 \%$ larger than the observed bow shock distances. Merka et al. (2005a) extended their earlier investigation using a larger dataset observed by IMP 8 over 27 years. Also, they included the model of Verigin et al. (2001b) and found that this model underestimated the distance to the bow shock by about $10 \%$ for average parameters. For large solar wind dynamic pressure values, however, the predictions of Verigin et al. (2001b) were almost correct. The predicting capabilities of the other models were about the same as found earlier by Merka et al. (2003). Merka et al. (2005b) were trying to improve the model of Peredo et al. (1995) by transforming the bow shock crossings to the Geocentric Plasma Ecliptic system (GPE defined by Merka and Szabo, 2004) where the X-axis points opposite to the solar wind direction and the ecliptic north is in the $\mathrm{X}-\mathrm{Z}$ plane. The GPE-based bow shock model was found to be more accurate and equally or more stable compared to the investigated other models.

Interplanetary plasma and magnetic field parameters significantly change in connection with large solar events like 
flares and coronal mass ejections (CMEs). The velocity of the solar wind can increase above $1500 \mathrm{~km} \mathrm{~s}^{-1}$, while the average value is $\sim 400 \mathrm{~km} \mathrm{~s}^{-1}$. Also, the plasma density can be about 10-20 times larger than average. High solar wind dynamic pressure compresses the terrestrial magnetosphere. Also, when the IMF $B_{\mathrm{Z}}$ is negative, the standoff distance of the magnetopause can significantly decrease as a result of the possible reconnection at the nose according to several studies (Sibeck et al., 1991; Petrinec and Russell, 1993; Roelof and Sibeck, 1993; Shue et al., 1997, 1998; Chao et al., 2002; Lin et al., 2010).

In this paper, three events will be discussed from the declining phase of the last solar cycle when one or more major CMEs were detected on the Sun. The extensive disturbances raised in the interplanetary plasma and field reached the Earth and caused extremely strong geomagnetic storms. During these events, the geosynchronous GOES satellites (at an orbit of 6.6 Earth radii, $R_{\mathrm{E}}$ ) observed the earthward displacement of the magnetopause. They spent several hours in the magnetosheath and measured magnetic field of interplanetary origin having a negative $B_{\mathrm{Z}}$ component. The Cluster spacecraft observed the bow shock and the magnetopause very close to the Earth in one event, and they crossed the bow shock at unusual locations several times in another case.

In Sect. 2, three magnetopause and four bow shock models will be presented which describe these discontinuities in considerably different ways. In Sect. 3, the three events will be investigated in detail; the observed locations of the magnetopause and the bow shock will be compared to the predictions of the selected models based on measured interplanetary parameters. In Sect. 4, a comparative analysis of the presented models will be performed in two special cases: (1) the IMF is extremely large and the solar wind dynamic pressure is above average, and (2) the solar wind dynamic pressure is very high and the IMF is average. The general discussion will include a comparison of plasma and field parameters provided by a global 3-D MHD model with data observed by Cluster 1 .

\section{Modelling the magnetopause and the bow shock}

The magnetopause and bow shock models which are used in this study are empirical models except for the bow shock model of Verigin et al. (2001a,b, 2003). The coefficients of the equations were determined from a great number of observations performed under different upstream conditions, and therefore the applied database influences the validity of the model. Two-dimensional magnetopause and bow shock models assume cylindrical symmetry around the aberrated SunEarth line, i.e. around the average solar wind direction (taking into account the aberration due to the Earth's rotation around the Sun). They provide relatively simple mathematical expressions for the size and shape of the discontinuity.
MP1, the two-dimensional magnetopause model of Shue et al. (1998), was selected based on the comparative studies of Yang et al. (2002) and Suvorova et al. (2005) investigating extreme solar wind conditions. It uses simple expressions for the standoff distance and for the flaring angle of the magnetopause, and its forecasting capability for magnetosheath encounters of geosynchronous satellites seemed to be satisfactory compared to the other investigated models. MP2, the three-dimensional magnetopause model by Lin et al. (2010), is taking into account the effect of the dipole tilt angle in addition to solar wind parameters (including extreme values), and it provided the smallest standard deviation for the magnetopause location when compared with other models. MP3 is a modified version of the two-dimensional magnetopause model by Verigin et al. (2009). It was selected since its approach for taking into account the effect of the IMF is different from the other two models. However, this model has not been tested previously for extreme solar wind conditions.

The comparative studies of Merka et al. (2003, 2005a,b) analyzed two of the bow shock models which will be used here for detailed investigations. BS2, the two-dimensional model of Farris and Russell (1994) combined with the model of Cairns et al. (1995), and BS4, the three-dimensional model of Verigin et al. (2001b), provided reliable predictions for the bow shock location. BS3, the three-dimensional model by Jerab et al. (2005), is an improved version of two earlier models (Formisano, 1979; Nemecek and Safrankova, 1991) which were included in the comparative studies of Merka et al. $(2003,2005 a, b)$. The arbitrary model BS1 uses a simple two-dimensional shape for the bow shock (Farris et al., 1991) combined with the standoff distance provided by Farris and Russell (1994).

\subsection{Applied magnetopause models}

Model 1 (MP1). The magnetopause model of Shue et al. $(1997,1998)$ applied the functional form

$R_{\mathrm{MP}}=R_{\mathrm{MP}}\left(\frac{2}{1+\cos \theta}\right)^{\alpha}$

where $R_{\mathrm{MP}}$ is the radial distance to the observation point and $\theta=\arccos \left[X / R_{\mathrm{MP}}\right]$ is the solar zenith angle, SZA. This form has two parameters: $R_{\mathrm{MP}_{0}}$ is the standoff distance and $\alpha$ controls the tail flaring. The parameters were determined as the function of the solar wind dynamic pressure $P_{\mathrm{d}}$ and IMF $B_{\mathrm{Z}}$ in Geocentric Solar Magnetospheric coordinates (where $X$ is the aberrated direction from the Earth to the Sun, and the magnetic dipole is in the $\mathrm{X}-\mathrm{Z}$ plane). The model of Shue et al. (1997) was improved by Shue et al. (1998) for extreme solar wind disturbances $\left(B_{\mathrm{Z}}<-18 \mathrm{nT}\right.$ and $\left.P_{\mathrm{d}}>8.5 \mathrm{nPa}\right)$ by introducing a nonlinear dependence of the parameters on solar wind conditions:

$R_{\mathrm{MP}_{0}}=\left\{10.22+1.29 \tanh \left[0.184\left(B_{\mathrm{z}}+8.14\right)\right]\right\} P_{\mathrm{d}}^{-\frac{1}{6.6}}$ 
and $\alpha=\left(0.58-0.007 B_{\mathrm{Z}}\right)\left(1+0.024 \ln \left[P_{\mathrm{d}}\right]\right)$ when $P_{\mathrm{d}}$ is in $\mathrm{nPa}$ and $B_{\mathrm{z}}$ in nT.

These formulas represent the saturation effects of $B_{\mathrm{Z}}$ on the standoff distance and the saturation effects of $P_{\mathrm{d}}$ on the flaring of the magnetopause. The two models from Shue et al. $(1997,1998)$ provide similar magnetopause distances for average solar wind parameters.

Model 2 (MP2). Lin et al. (2010) developed an asymmetric three-dimensional magnetopause model in aberrated GSM coordinates in which the location and shape of the magnetopause depends not only on $\theta$ solar zenith angle, but also on the azimuth angle $\varphi=\arctan \left[Z_{\mathrm{GSM}}, Y_{\mathrm{GSM}}\right]$ and the dipole tilt angle $\phi$ measured from $+Z_{\mathrm{GSM}}$ towards the solar direction. From the interplanetary parameters, the magnetic pressure $P_{\mathrm{m}}$ is also taken into account (under average conditions $\left.P_{\mathrm{m}}<P_{\mathrm{d}} / 100\right)$ in addition to solar wind dynamic pressure $P_{\mathrm{d}}$ and IMF $B_{Z}$ :

$$
\begin{aligned}
R_{\mathrm{MP}}(\theta, \varphi, \phi)= & R_{\mathrm{MP}_{0}} F(\theta, \varphi, \phi)+a_{14}\left(P_{\mathrm{d}}+P_{\mathrm{m}}\right)^{a_{15}} \\
& \left(\exp \left[d_{\mathrm{n}} \psi_{\mathrm{n}}^{a_{21}}\right]+\exp \left[d_{\mathrm{s}} \psi_{\mathrm{s}}^{a_{21}}\right]\right)
\end{aligned}
$$

where

$R_{\mathrm{MP}_{0}}=a_{0}\left(P_{\mathrm{d}}+P_{\mathrm{m}}\right)^{a_{1}}\left(1+a_{2} \frac{\exp \left[a_{3} B_{\mathrm{z}}\right]-1}{\exp \left[a_{4} B_{\mathrm{z}}\right]+1}\right)$

and

$F(\theta, \varphi, \phi)=\left\{\cos (\theta / 2)+a_{5} \sin (2 \theta)(1-\exp [-\theta])\right\}^{\beta(\varphi, \phi)}$.

The first term in Eq. (2) corresponds to Eq. (1) of model MP1; here $\beta=(\varphi, \phi)$ controls the tail flaring (see Eq. 2a). The second term represents the influence of the polar indentation vertices where $\psi_{\mathrm{n}}\left(\psi_{\mathrm{s}}\right)$ is the angle between the direction of $R_{\mathrm{MP}}(\theta, \varphi, \phi)$ and the direction of the northern (southern) indentation vertex, while $d_{\mathrm{n}}$ and $d_{\mathrm{s}}$ depend on the dipole tilt angle $\phi$.

The complete set of equations of Lin et al. (2010) contains 22 fitting coefficients $a_{i}(i=0,21$, nine of them are explicitly given here in Eqs. (2) and (2a), which were determined from about 2500 magnetopause crossings observed by more than 10 different spacecraft using the Levenberg-Marquart method for nonlinear multiparameter fitting. Extreme values of solar wind parameters (like $B_{\mathrm{Z}}=-49.6 \mathrm{nT}$ and $P_{\mathrm{d}}=33.7 \mathrm{nPa}$ ) were also represented in the database. More than half of the magnetopause crossings (the 1482 cases observed by Hawkeye) were used only for fitting the indentations. According to Lin et al. (2010), their model improved the prediction capability of describing the three-dimensional structure of the magnetopause compared with eight previous empirical models, especially in the cusp regions.

Model 3 (MP3). Verigin et al. (2009) developed an empirical cylindrical model using almost 3000 magnetopause crossings observed by the Prognoz satellites and by the Interball 1 satellite, which were collected mainly under average interplanetary conditions. For the shape of the magnetopause, they used an expression originally suggested by Howe and Binsack (1972):

$X(Y)=R_{\mathrm{MP}_{0}}-\frac{D^{2}}{2 \pi^{2} R_{C}} \tan ^{2}\left(\frac{\pi Y}{D}\right)$,

where $X$ is the distance from the center of the Earth in the aberrated solar direction and $Y$ is perpendicular to it; $R_{\mathrm{MP}_{0}}$ is the standoff distance; $R_{\mathrm{C}}$ is the magnetopause curvature radius in the subsolar region; and $D$ is the magnetotail diameter at a large distance from the Earth $(X \rightarrow-\infty)$. The parameters were determined by minimizing the root mean square deviation of the observed magnetopause positions from the surface described by Eq. (3). Verigin et al. (2009) found that $R_{\mathrm{MP}_{0}}, R_{\mathrm{C}}$, and $D$ depend only on solar wind dynamic pressure, and the influence of the IMF could be neglected.

In order to include the effect of large interplanetary magnetic field values in this study, the model of Verigin et al. (2009) is modified here so that it takes into account the pressure of the compressed magnetosheath field in addition to the solar wind dynamic pressure when determining the shape and size of the magnetopause. Crooker et al. (1982) provided an empirical formula for the magnetosheath field at the stagnation point as

$B_{\mathrm{SH}}=\sqrt{4 B_{\mathrm{SW}}^{t} B_{\mathrm{ST}}}$,

where $B_{\mathrm{ST}}$ is the magnetospheric stagnation field, while the shocked solar wind is approximated by $4 B_{\mathrm{SW}}^{t}$. The IMF component transverse to the solar wind flow is $B_{\mathrm{SW}}^{t}=$ $\sqrt{\left(B_{\mathrm{SW}}^{y}\right)^{2}+\left(B_{\mathrm{SW}}^{z}\right)^{2}}$.

In this new model, the stagnation field pressure is balanced by the total plasma and magnetic pressure in the magnetosheath as

$P=B_{\mathrm{ST}}^{2} /(8 \pi)=k P_{\mathrm{d}}+B_{\mathrm{SH}}^{2} /(8 \pi)$,

where $k=0.881$ tells how much the plasma pressure diminished from the upstream solar wind to the magnetopause (cf. Spreiter et al., 1966; Howe and Binsack, 1972). When substituting $B_{\mathrm{SH}}$ from Eq. (3a) to Eq. (3b), $B_{\mathrm{ST}}$ can be calculated from a quadratic equation providing $B_{\mathrm{SH}}$, and $P$ can be determined as

$P=k P_{\mathrm{d}}\left(1+\delta+\sqrt{\delta^{2}+2 \delta}\right)$ where $\delta=\left(B_{\mathrm{SW}}^{t}\right)^{2} /\left(\pi k P_{\mathrm{d}}\right)$.

The fitting values for $R_{\mathrm{MP}}, R_{\mathrm{C}}$, and $D$ in Eq. (3) were determined from the magnetopause crossings used by Verigin et al. (2009) by minimizing the rms deviation between observations and the theoretical surface. The following expressions were provided for the parameters:

$R_{\mathrm{MP}_{0}}=11.16 R_{\mathrm{E}} P^{-1 / 6}$, 
$R_{\mathrm{C}}=16.51 R_{\mathrm{E}} P^{-1 / 6}$, and

$D=98.06 R_{\mathrm{E}} P^{-1 / 6}$.

In this model the effect of the interplanetary magnetic field is taken into account through the corrected pressure (see Eqs. 3c and 3d).

\subsection{Applied bow shock models}

Model 1 (BS1). The simple empirical two-dimensional model of Farris et al. (1991) described the bow shock using the functional form

$$
R_{\mathrm{BS}}=R_{\mathrm{BS}_{0}} \frac{1+\varepsilon}{1+\varepsilon \cos \theta},
$$

where $\theta$ is the solar zenith angle in aberrated coordinates, $R_{\mathrm{BS}_{0}}$ is the standoff distance, and $\varepsilon$ is the eccentricity. Farris et al. (1991) received $\varepsilon=0.81$ and $R_{\mathrm{BS}_{0}}=13.7 R_{\mathrm{E}}$ when fitting this formula to 351 independent bow shock crossings observed by the ISEE 1 spacecraft.

Model 1 of the present study is using Eq. (4) with $\varepsilon=0.81$ as determined by Farris et al. (1991) for describing the shape of the bow shock. $R_{\mathrm{BS}_{0}}$ standoff distance is calculated from the actually measured interplanetary solar wind and magnetic field parameters according to Farris and Russell (1994):

$R_{\mathrm{BS}_{0}}=R_{\mathrm{MP}_{0}}\left(1+1.1 \frac{(\gamma-1) M_{\mathrm{ms}}^{2}+2}{(\gamma+1)\left(M_{\mathrm{ms}}^{2}-1\right)}\right)$,

where $M_{\mathrm{ms}}=V_{\mathrm{sw}} / V_{\mathrm{ms}}$ is the upstream magnetosonic Mach number, $\gamma$ polytropic index is $5 / 3$, and the magnetopause standoff distance $R_{\mathrm{MP}_{0}}$ is taken from the model of Lin et al. (2010).

Model 2 (BS2). The bow shock standoff distance provided by Farris and Russell (1994) can be combined with other representations of the whole bow shock surface. Model 2 uses the formula given by Cairns et al. (1995)

$X=R_{\mathrm{BS}_{0}}-0.0223\left(P_{\mathrm{d}} / 1.8\right)^{1 / 6} Y^{2} R_{\mathrm{E}}{ }^{-1}$,

where $X$ is the distance from the center of the Earth in the aberrated solar direction and $Y$ is perpendicular to it. The magnetopause standoff distance $R_{\mathrm{MP}_{0}}$ in Eq. (4a), providing $R_{\mathrm{BS}_{0}}$ for Eq. (4b), is taken from the model of Shue et al. (1998). Model BS2 was analyzed in the comparative studies of Merka et al. (2003, 2005a,b).

Model 3 (BS3). Jerab et al. (2005) improved the threedimensional empirical model which was first presented by Formisano (1979) and later modified by Nemecek and Safrankova (1991). For the bow shock surface, Jerab et al. (2005) used the formula

$R_{\mathrm{BS}}=\frac{R_{\mathrm{av}}}{R_{0}} \frac{C}{P_{\mathrm{d}}^{1 / 6}}\left(1+D \frac{(\gamma-1) M^{2}+2}{(\gamma+1)\left(M^{2}-1\right)}\right)$, where $D=0.937(0.846+0.042|B|)$ and $M=M_{\mathrm{A}}$. C is a constant and $R_{0}$ is the geocentric distance to the subsolar point of the average bow shock position which is described in unaberrated Geocentric Solar Equatorial (GSE) coordinates as

$$
\begin{aligned}
R_{\mathrm{av}}= & a_{11} X^{2}+a_{22} Y^{2}+a_{33} Z^{2}+a_{12} X Y+a_{14} X \\
& +a_{24} Y+a_{34} Z+a_{44} .
\end{aligned}
$$

Formisano (1979) took $a_{34}=0$, neglecting the $Z$ dependence in Eq. (5a). The coefficients $a_{i j}$ providing the best fit were determined from the observations; Formisano (1979) used about 2500 bow shock crossings (mainly from the HEOS 2 spacecraft), while Jerab et al. (2005) used about 5400 crossings (observed by 5 spacecraft on different orbits) as presented in their papers. Two earlier versions of model BS3 (Formisano, 1979; Nemecek and Safrankova, 1991) were investigated in the comparative studies of Merka et al. (2003, 2005a,b).

Model 4 (BS4). Verigin et al. (2001a,b, 2003) developed a three-dimensional semi-empiric bow shock model combined with MHD solution using the GIPM (Geocentric InterPlanetary Medium) reference frame where the $\mathrm{X}$-axis is antiparallel to the solar wind direction and the Y-axis is parallel (if $B_{\mathrm{x}}<0$ ) or anti-parallel (if $B_{\mathrm{x}}>0$ ) to the IMF component perpendicular to the solar wind direction, meaning that $B_{\mathrm{Z}}=0$. In the GIPM coordinate system, the IMF points to the second or fourth quadrant of the $\mathrm{X}-\mathrm{Y}$ plane, i.e. the quasi-parallel region of the bow shock can be separated from the quasi-perpendicular side. As the IMF is expected to influence the size and shape of the bow shock, the GIPM frame provides an easier comparison of different observations.

The equation for the quasi-hyperbolic bow shock surface can be written in the following form (cf. Verigin et al., 2001a,b)

$$
\begin{aligned}
X= & R_{\mathrm{BS}_{0}}+\chi R_{\mathrm{S}}\left(M_{\mathrm{as}}^{2}-1\right)-\frac{1}{2}(1-\chi) \\
& \sqrt{\left(M_{\mathrm{as}}^{2}-1\right)\left(Y^{2}+Z^{2}\right)}-\chi R_{\mathrm{S}}\left(M_{\mathrm{as}}^{2}-1\right) \\
& \sqrt{1-\frac{(1-\chi)}{\chi R_{\mathrm{S}}} \sqrt{\frac{Y^{2}+Z^{2}}{M_{\mathrm{as}}^{2}-1}}+\frac{(1+\chi)^{2}\left(Y^{2}+Z^{2}\right)}{4 \chi^{2} R_{\mathrm{S}}^{2}\left(M_{\mathrm{as}}^{2}-1\right)}},
\end{aligned}
$$

where the standoff distance of the bow shock $R_{\mathrm{BS}}$ and its curvature radius at the nose $R_{\mathrm{S}}$ can be determined from parameters $R_{\mathrm{MP}_{0}}$ and $R_{\mathrm{C}}$ for the magnetopause (see Eqs. 3 and $3 \mathrm{~d}$ ) and from the compression ratio of the solar wind flow as described by Verigin et al. (2001a,b). In Eq. (6), $\chi=3.2 /\left(M_{\mathrm{as}}+1\right)$ is the shaping parameter where $M_{\mathrm{as}}=$ $1 / \sin \vartheta_{\text {as }}$ is a function of the asymptotic cone angle $\vartheta_{\text {as }}$ of the tail slope (Verigin et al., 2001a,b). In this work, magnetopause parameters $R_{\mathrm{MP}_{0}}$ and $R_{\mathrm{C}}$ provided by the new model MP3 in Eq. (3d) are used. Verigin et al. (2001a,b, 2003) used the parameters $R_{\mathrm{MP}_{0}}$ and $R_{\mathrm{C}}=2 R_{\mathrm{MP}_{0}} /(2-\alpha)$ 


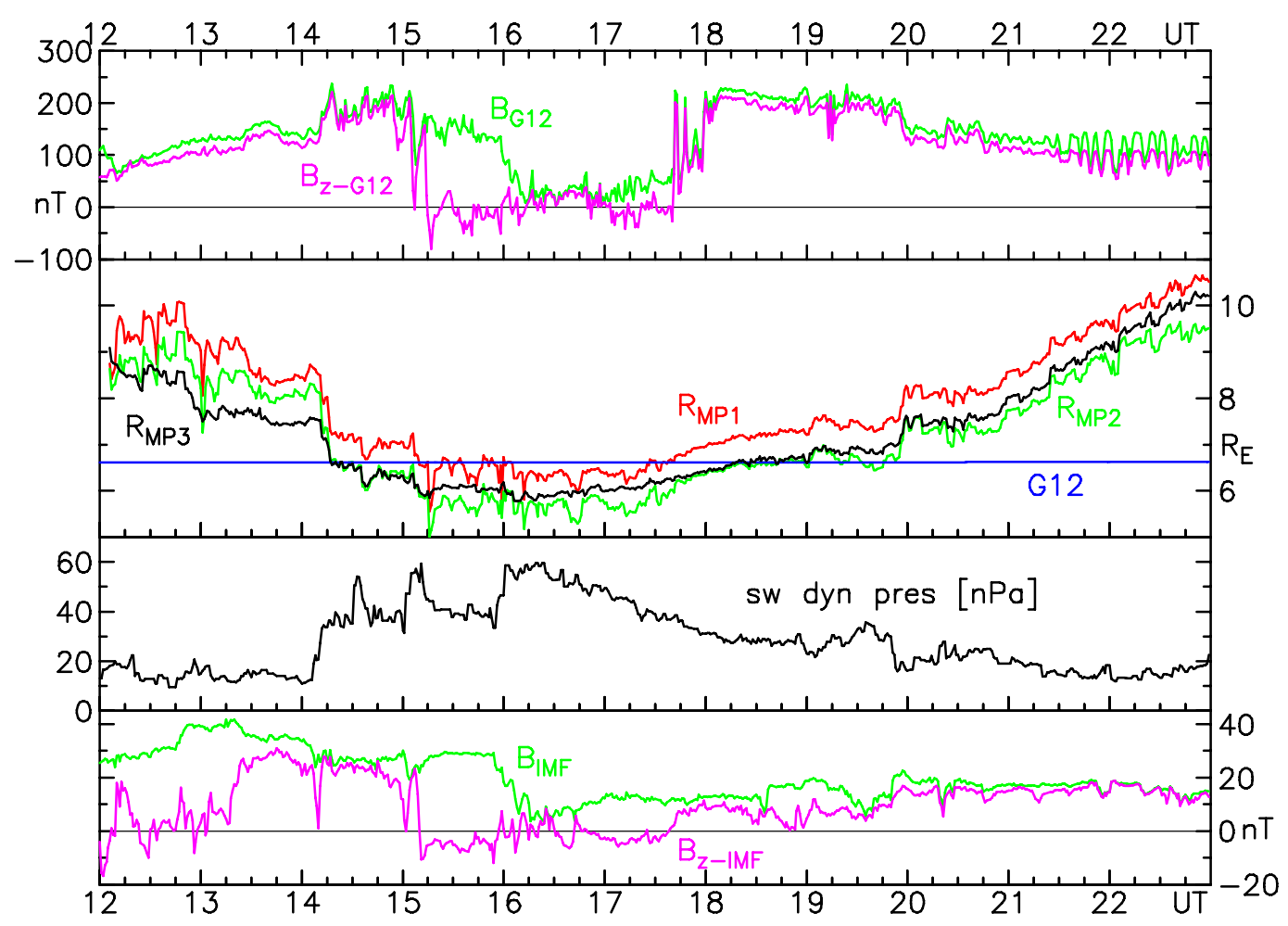

Fig. 1. 17 January 2005. Top panel: GOES 12 magnetic field observations: total value (green line), and $B_{\mathrm{Z}}$ in GSM coordinates (lilac). Panel 2: model magnetopause locations for angular directions corresponding to GOES 12 position: MP1 in red (Shue et al., 1998), MP2 in green (Lin et al., 2010), and MP3 in black (new model); G12 trajectory: blue line. Panel 3: solar wind dynamic pressure. Bottom panel: IMF: total field (green), and $B_{\mathrm{Z}}$ (lilac). Interplanetary data from Wind measurements.

from the model of Shue et al. (1998), presented here as MP1. This earlier version of model BS4 was analyzed in the comparative studies of Merka et al. (2005a,b).

\section{Case studies}

In the following analysis, 1-min-resolution interplanetary parameters are used which were taken from the OMNI dataset available at NASA's Coordinated Data Analysis Web (CDAWeb): http://cdaweb.gsfc.nasa.gov/istp_public/. The OMNI dataset provides plasma and magnetic field data measured by different spacecraft upstream of the terrestrial bow shock and propagated to the nose of the bow shock. The interplanetary parameters were determined from Wind measurements for 17 January 2005 and from ACE measurements for 31 October 2003 and for 20 November 2003. 1-minresolution magnetic field data from the Geostationary Operational Environmental Satellites are also available at NASA's CDAWeb. GOES 10 and GOES 12 were collecting magnetic field data at the time of the investigated cases. There was no low energy plasma detector aboard the GOES satellites, energetic particle data were provided only for GOES 10 on 20 November 2003. 4-s-resolution magnetic field data measured by the FGM instrument (Balogh et al., 2001), proton velocity and density measured by the two instruments CODIF and HIA of the Cluster Ion Spectrometry experiment (Rème et al., 2001) aboard the Cluster spacecraft were taken from the Cluster Hungarian Data Centre, available at http://hdc.rmki.kfki.hu/ $\mathrm{cdms} /$. They were in good agreement with the data available at the Cluster Active Archive at http://caa.estec.esa.int/.

\subsection{January 2005}

As a result of a large CME, solar wind velocity and density significantly increased upstream of the terrestrial bow shock on 17 January 2005. The two lower panels of Fig. 1 present the solar wind dynamic pressure and the IMF (total value and $B_{\mathrm{Z}}$ component) taken from NASA's OMNI dataset between 12:00 and 23:00 UT on 17 January 2005. The dynamic pressure was extremely high between 14:00 and 20:00 UT. The IMF was large only until about 16:00 UT and negative (but not extremely large) $B_{\mathrm{z}}$ values were measured most of the time between 15:10 and 17:40 UT. Magnetic field data (total value and the $B_{\mathrm{Z}}$ component) measured aboard GOES 12 are shown in the top panel of Fig. 1. The negative or very small $B_{\mathrm{Z}}$ values observed by GOES 12 between 15:15 UT and 17:41 UT indicate that the satellite was in the magnetosheath 
as it was close to the Sun-Earth line. Also, the variations in $B_{\mathrm{z}}$ (lilac line) and in the total values (green) are in good agreement with the variations of the IMF. However, the magnetic field trends observed by GOES 12 are similar to those of the IMF also before 15:15 UT and after 17:41 UT for some time, but $B_{\mathrm{Z}}$ is positive. Since no plasma data are available from GOES 12, it is difficult to tell the exact times when the satellite crossed the magnetopause during the time intervals 15:00-15:15 UT and 17:41-18:00 UT. Panel 2 of Fig. 1 presents the location of the magnetopause for angular directions corresponding to the GOES 12 position as calculated from the three different models presented in Sect. 2: MP1 (Shue et al., 1998) in red, MP2 (Lin et al., 2010) in green, and MP3 (new model) in black. The orbit of GOES 12 at $6.62 R_{\mathrm{E}}$ is marked by a blue line. For the model calculations, the propagating time of the solar wind from the bow shock to GOES 12 was taken into account based on the measured upstream solar wind velocity and on the estimated flow time of plasma packages from the bow shock to the position of the satellite (cf. Tátrallyay and Erdős, 2002).

As seen in Fig. 1, model MP1 (Shue et al., 1998) predicts that the satellite is upstream of the magnetopause between 15:05 UT and 17:41 UT when $B_{\mathrm{Z}}$ is negative or close to zero, except for the large fluctuation around 15:10 UT. Model MP2 (Lin et al., 2010) and the new model MP3 provide longer excursion to the magnetosheath, but the difference between the predicted magnetopause locations and the satellite's orbit is small (within $0.2 R_{\mathrm{E}}$ ) for about $1 \mathrm{~h}$ before 15:05 UT and for more than $1 \mathrm{~h}$ after 17:41 UT. Figure 2 shows the trajectory of GOES 12 on 17 January 2005 in cylindrical coordinates (G12 gray line); the section in the magnetosheath (between 15:15 and 17:41 UT) is marked in lilac. The solid red curve presents the location of the magnetopause calculated from model MP1 (Shue et al., 1998) with parameters around 17:30 UT. The bow shock profile shown by the solid blue line was calculated with the same interplanetary parameters using model BS2 (Cairns et al., 1995; Farris and Russell, 1994). For comparison, dashed curves present the magnetopause and the bow shock for average solar wind parameters.

Figure 2 also presents the inbound pass of Cluster 1 (C1) between 08:00 UT and 24:00 UT in cylindrical coordinates. Sections of the orbit are shown in lilac when the satellite was in the magnetosheath according to magnetic field and plasma measurements. Cluster 1 was in the magnetosheath between 10:25 and 10:42 UT, as seen in Fig. 3 from the sudden changes in the measured parameters. Figure 3 shows the velocity $V$, magnetic field $B$, and number density $N$ data measured aboard Cluster 1 in red and Cluster 4 in green. Plasma parameters were provided by CIS/HIA for Cluster 1 and by CIS/CODIF for Cluster 4. Black traces show the results of a global 3-D MHD model which will be discussed in Sect. 4. Velocity decreased while magnetic field increased aboard Cluster 1 for 17 mins when the spacecraft was in the magnetosheath. The number density values for Cluster 1 are decreasing (instead of increasing) in the magnetosheath in-

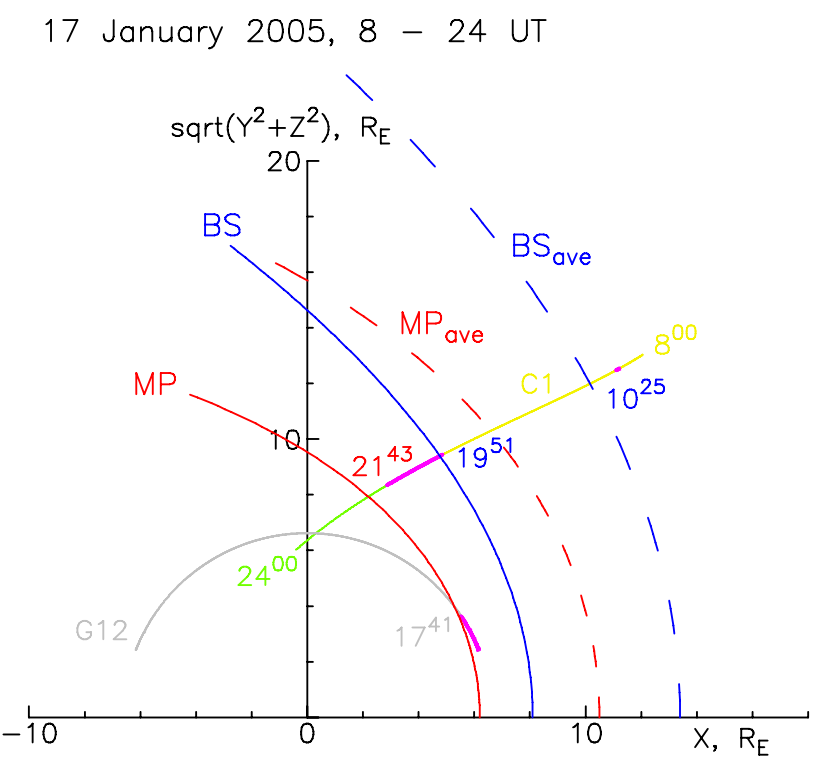

Fig. 2. Cluster 1 inbound trajectory and GOES 12 orbit in cylindrical coordinates. Sections in the magnetosheath are marked in lilac. Yellow sections: in interplanetary field. Green line for $\mathrm{C} 1$, gray line for G12: in magnetosphere. BS2 model (Cairns et al., 1995; Farris and Russell, 1994) bow shock is shown by blue line and MP1 model (Shue et al., 1998) magnetopause by red line: dashed curves for average solar wind parameters, solid curves calculated with parameters around 17:40 UT when G12 reentered the magnetosphere. Times of bow shock and magnetopause crossings are given.

terval as the HIA detector was in solar wind mode detecting ions only from the solar direction, but the sudden change at the in- and outbound bow shock crossing can be seen. Green traces show the change of the parameters measured aboard Cluster 4, which observed multiple bow shock crossings (two excursions into the magnetosheath) indicating that the bow shock surface was between the two spacecraft (separated by about $1500 \mathrm{~km}$ ) for a few minutes. The number density was obviously increasing in the magnetosheath according to Cluster 4 data, namely two peaks were observed corresponding to the two magnetosheath intervals.

The next inbound bow shock crossing was about $7 R_{\mathrm{E}}$ closer to the Earth at 19:51 UT when solar wind dynamic pressure was decreasing after its peak value and the magnetosphere was expanding. Figure 3 presents magnetic field and plasma data also for the time interval from 18:15 to 23:00 UT. Unfortunately, plasma moments are unreliable before 19:51 UT when the velocity values are not presented and the proton number densities may be incorrect as the instruments were not in the proper operation mode. (Data Caveats are provided at http://cluster.cesr.fr:8000/ for the different operation modes.) The sudden increase in the magnetic field and number density data measured aboard both Cluster 1 and Cluster 4 indicate the time of the bow shock crossing as shown in Fig. 3. The sudden decrease in the velocity of 

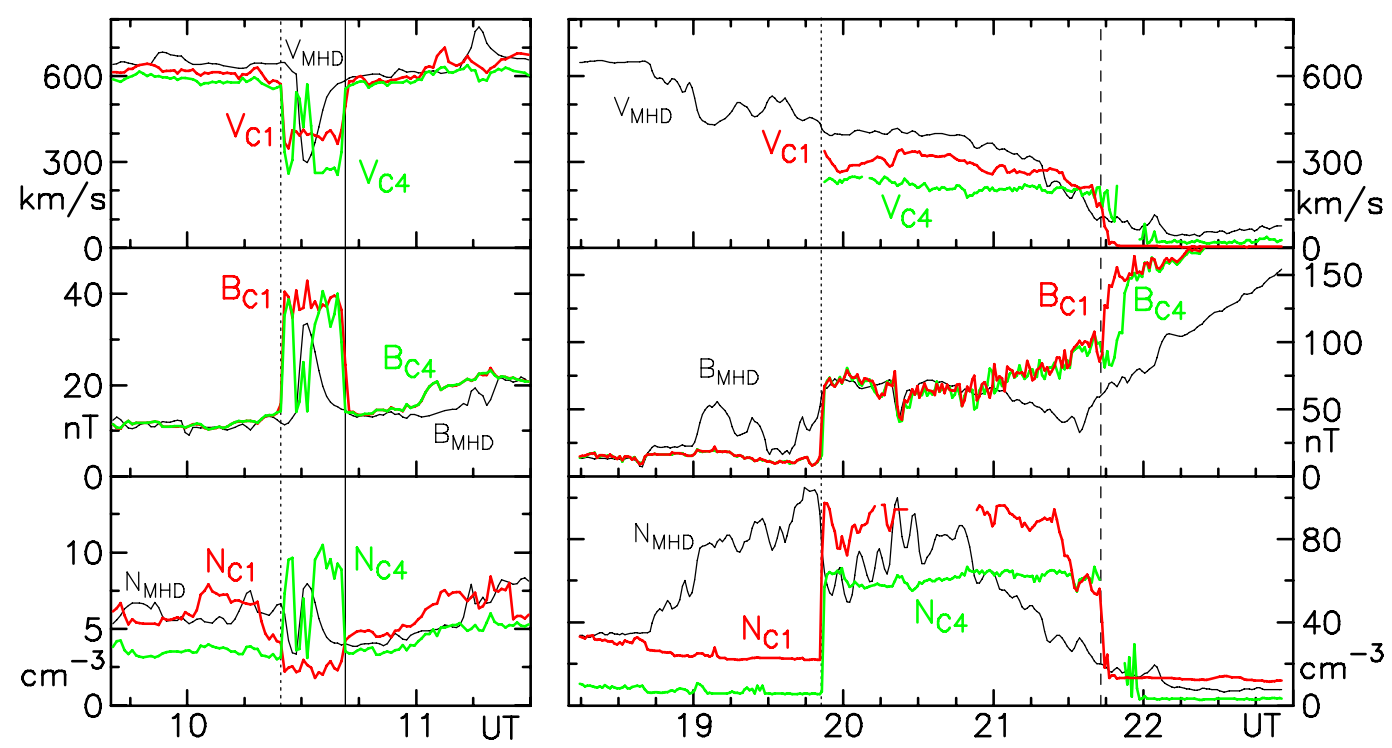

Fig. 3. 17 January 2005. Solar wind velocity $V$, magnetic field $B$, and proton number density $N$ measured by Cluster 1 in red and by Cluster 4 in green. The same parameters for the trajectory of Cluster 1 provided by a global 3-D MHD model run in CCMC (black curves). Observed inbound bow shock crossings at 10:25 and 19:51 UT marked by dotted lines; outbound crossing at 10:42 UT shown by solid line; inbound magnetopause crossing at 21:43 UT marked by dashed line.

electrons measured by the PEACE instrument (not shown here) also confirms that the bow shock was crossed by both spacecraft at 19:51 UT. Magnetic field is suddenly increasing when the magnetopause is crossed by Cluster 1 at 21:43 UT and by Cluster 4 a few minutes later. Velocity and number density are decreasing at the same time. In Fig. 2, the trajectory of Cluster 1 is marked in yellow when it was in the interplanetary field, and the green section was spent in the magnetosphere.

Figure 4 presents the results of different models providing the bow shock (top panel) and magnetopause location (second panel) for angular directions corresponding to the Cluster 1 position. The observed magnetopause location at 21:43 UT is in good agreement with the prediction of models MP2 (Lin et al., 2010) and MP3 (new model), as seen in panel 2. The magnetopause is farther away from the Earth according to model MP1 (Shue et al., 1998). The top panel shows that the best prediction for the bow shock is given by BS1 (Farris et al., 1991; Farris and Russell, 1994). The other models predict the bow shock at a distance of about $0.5-1 R_{\mathrm{E}}$ farther upstream than it was observed at 19:51 UT. Neither of the models predict the observed in- and outbound bow shock crossing at 10:25 and 10:42 UT, respectively. According to model BS3 (Jerab et al., 2005), however, Cluster 1 was supposed to observe multiple bow shock crossings around 13:00-14:00 UT.

\subsection{November 2003}

During the event on 20 November 2003, the interplanetary magnetic field had an extreme $B_{\mathrm{z}}<-30 \mathrm{nT}$ value for almost
$5 \mathrm{~h}$, and it was negative for more than $12 \mathrm{~h}$, as seen in Fig. 5. Therefore, entry to the magnetosheath by a dayside geosynchronous satellite is obviously indicated by observing negative $B_{\mathrm{Z}}$ values. Two GOES satellites were on the dayside when the solar wind dynamic pressure and the IMF reached large values. GOES 12 spent more than $6 \mathrm{~h}$; GOES 10 spent $5.5 \mathrm{~h}$ continuously upstream of the magnetopause.

The top panel and the second panel from the bottom of Fig. 5 present magnetic field observations of GOES 10 and GOES 12 , respectively (total value in green and $B_{\mathrm{Z}}$ in lilac in GSM coordinates). The panels to be found under the GOES measurements (panel 2 for GOES 10, bottom panel for GOES 12) show magnetopause locations predicted by different models for angular directions of the satellites: MP1 (Shue et al., 1998) in red, MP2 (Lin et al., 2010) in green, and MP3 (new model) in black. Solar wind dynamic pressure and the IMF are provided in the two middle panels. For the model calculations, the propagating time of the solar wind from the bow shock to the GOES satellites was taken into account as described in Sect. 3.1. The times of observed outbound magnetopause crossings are marked by solid vertical lines; inbound crossings are marked by dashed lines. The negative $B_{\mathrm{Z}}$ values observed by GOES 10 before 14:00 UT and by GOES 12 after 21:30 UT are not considered as magnetopause crossings as the satellites were close to the terminator or behind it.

Figure 5 shows that MP1 (Shue et al., 1998) underestimates the displacement of the magnetopause towards the Earth. It predicts that the excursion to the magnetosheath of both satellites was shorter than observed (by about $2 \mathrm{~h}$ for 


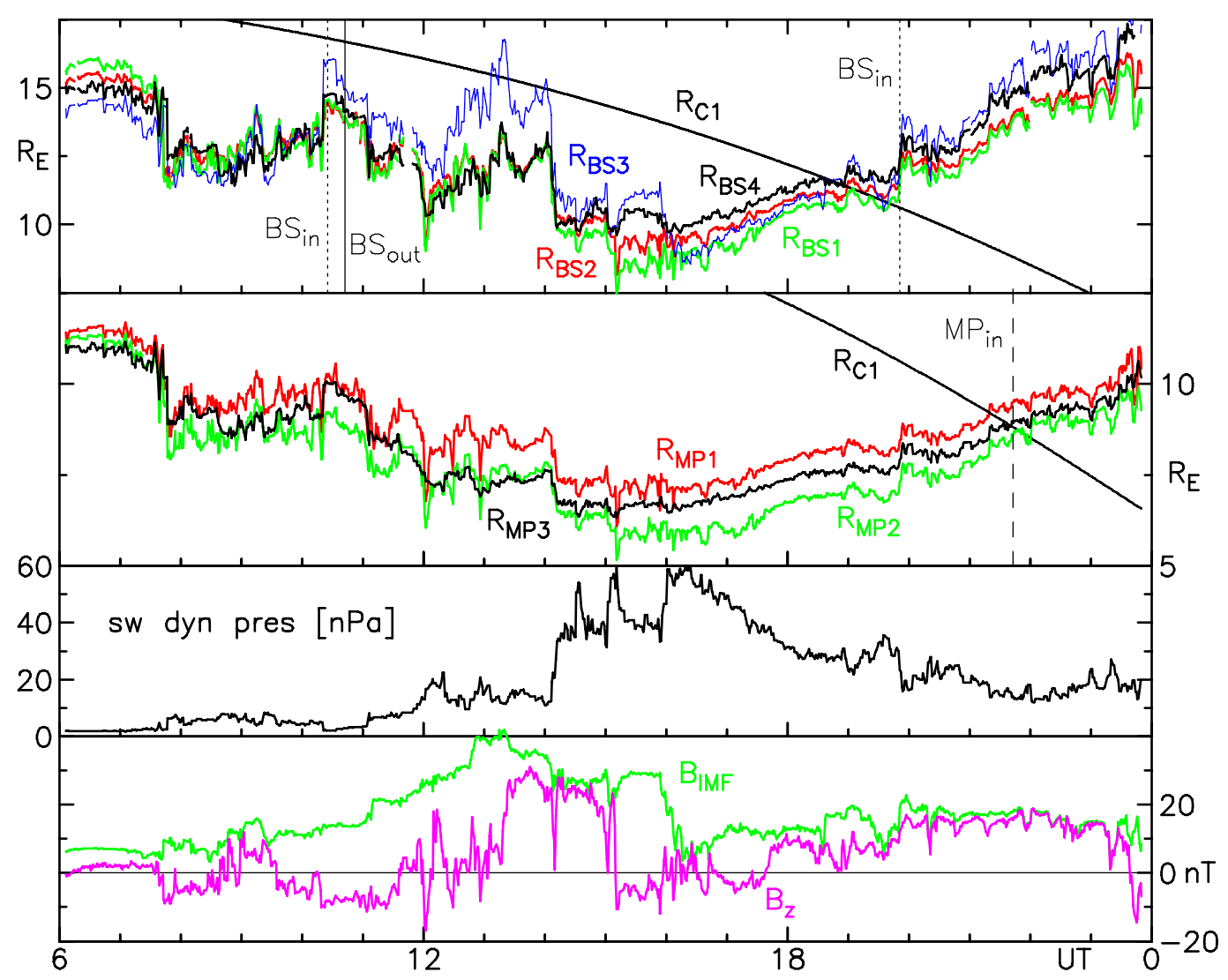

Fig. 4. 17 January 2005. Top panel: model bow shock locations for angular directions corresponding to Cluster 1 position $\left(R_{\mathrm{C} 1}\right.$ black line): BS1 in green (Farris et al., 1991; Farris and Russell, 1994), BS2 in red (Cairns et al., 1995; Farris and Russell, 1994), BS3 in blue (Jerab et al., 2005), and BS4 in black (Verigin et al., 2001a,b, 2003). Observed bow shock crossings are marked by vertical lines (inbound dotted, outbound solid) at 10:25, 10:42, and 19:51 UT. Panel 2: model magnetopause locations for Cluster 1 angular directions: MP1 in red (Shue et al., 1998), MP2 in green (Lin et al., 2010), and MP3 in black (new model). Observed magnetopause crossing at 21:43 UT is marked by dashed line. Panel 3: solar wind dynamic pressure. Bottom panel: IMF total value (green) and $B_{\mathrm{Z}}$ (lilac). Interplanetary data from Wind measurements.

GOES 10 , by more than $3 \mathrm{~h}$ for GOES 12). Models MP2 (Lin et al., 2010) and MP3 (new model) provide better agreement with the observations; the predictions of MP3 are better at the beginning of the long magnetosheath interval for both satellites, while the observed re-entry time to the magnetosphere is better predicted by MP2 for GOES 12. The three models provide almost the same location for the magnetopause for the GOES 10 angular directions around the inbound crossing between 20:00 and 22:00 UT. MP3 also predicts the two short magnetosheath intervals for GOES 12 at 13:02-13:15 UT and at 13:27-14:02 UT; MP2 predicts part of them, while MP1 does not predict them. Neither of the models provides magnetopause crossings for GOES 10 at 16:31 and 16:42 UT, but the prediction of MP3 is closest to the geosynchronous orbit.

\subsection{October 2003}

On 29-31 October 2003 (Halloween storm), several large solar events were observed which were followed by large geomagnetic storms. Due to the extreme disturbances in interplanetary space, there are long gaps in the OMNI dataset between 05:49 UT on 29 October and 11:14 UT on 31 October. Plasma and field parameters (based on Geotail measurements) are provided only for about $5 \mathrm{~h}$ in the evening of 29 October. From the ACE spacecraft, the velocity and density of the alpha particles and the IMF components are available for all 3 days showing large disturbances. However, proton density data are provided only from about 11:00 UT on 31 October, while proton velocity data are available from about 01:00 UT.

Both GOES 10 and GOES 12 were on the dayside in the afternoon hours of Universal Time, and they spent several hours in the magnetosheath (observing negative $B_{\mathrm{Z}}$ ) on 29 and 30 October when the magnetosphere was compressed 


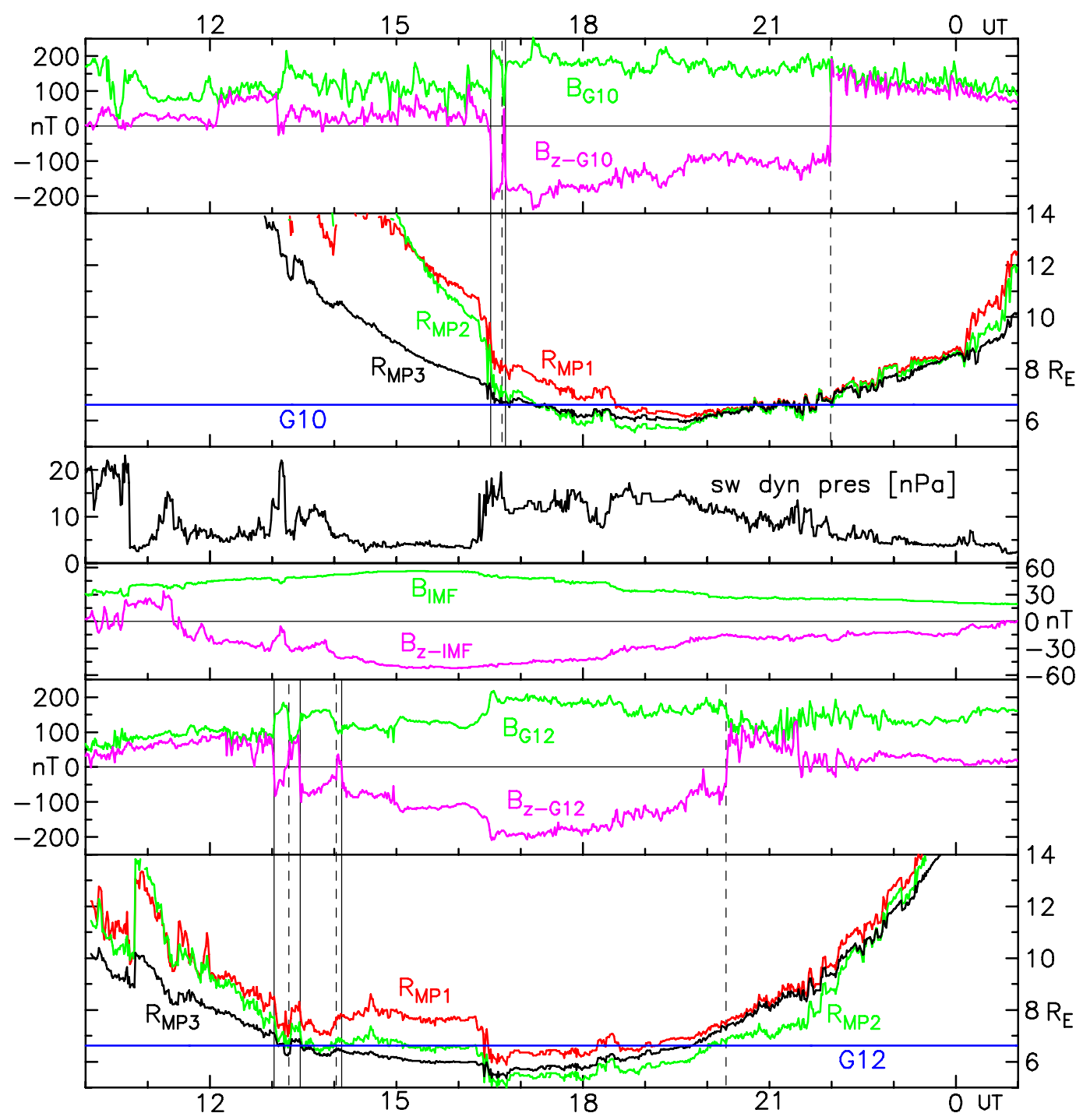

Fig. 5. 20 November 2003. Top panel: GOES 10 magnetic field observations: total value (green) and $B_{\mathrm{Z}}$ in GSM coordinates (lilac). Panel 2: model magnetopause locations for angular directions of GOES 10 position: MP1 in red (Shue et al., 1998), MP2 in green (Lin et al., 2010), and MP3 in black (new model); G10 trajectory: blue line. Panel 3: solar wind dynamic pressure. Panel 4: IMF total value (green) and $B_{\mathrm{Z}}$ (lilac). Panel 5: GOES 12 magnetic field: total value (green) and $B_{\mathrm{Z}}$ (lilac). Bottom panel: model magnetopause locations for angular directions of GOES 12 position: MP1 in red (Shue et al., 1998), MP2 in green (Lin et al., 2010), and MP3 in black (new model); G12 trajectory: blue line. Solid vertical lines mark the time of observed outbound magnetopause crossings; dashed lines mark inbound crossings. Interplanetary data from ACE measurements.

supposedly due to the large dynamic pressure accompanied by large negative values of IMF $B_{\mathrm{Z}}$ (values below $-20 \mathrm{nT}$ ). In spite of the insufficient interplanetary data coverage, these events were discussed in several papers (e.g. Dmitriev et al., 2005; Lopez et al., 2007). Some earlier results will be presented later in this section, but GOES observations are not further analyzed in this work.

The four Cluster spacecraft were in the tail region on 29 30 October. On 31 October, however, they observed several out- and inbound bow shock and magnetopause crossings between 05:00 and 12:00 UT on their inbound path on the flanks. The Cluster satellites were upstream of the bow shock from 05:33 UT for about $10 \mathrm{~min}$, and around 11:37 UT and 11:57 UT for about $1 \mathrm{~min}$ in both cases as marked by blue crosses in Fig. 6 where the trajectory of Cluster 1 (C1) is shown from 00:00 to 22:00 UT. Sections in the magnetosheath are marked in lilac. The spacecraft made four excursions to the magnetosphere between 09:52 and 10:46 UT as shown in Fig. 7 and also indicated by a thin green trace around 10:15 UT in Fig. 6.

Figure 7 presents the total magnetic field, proton velocity and density for two time intervals on 31 October 2003. 


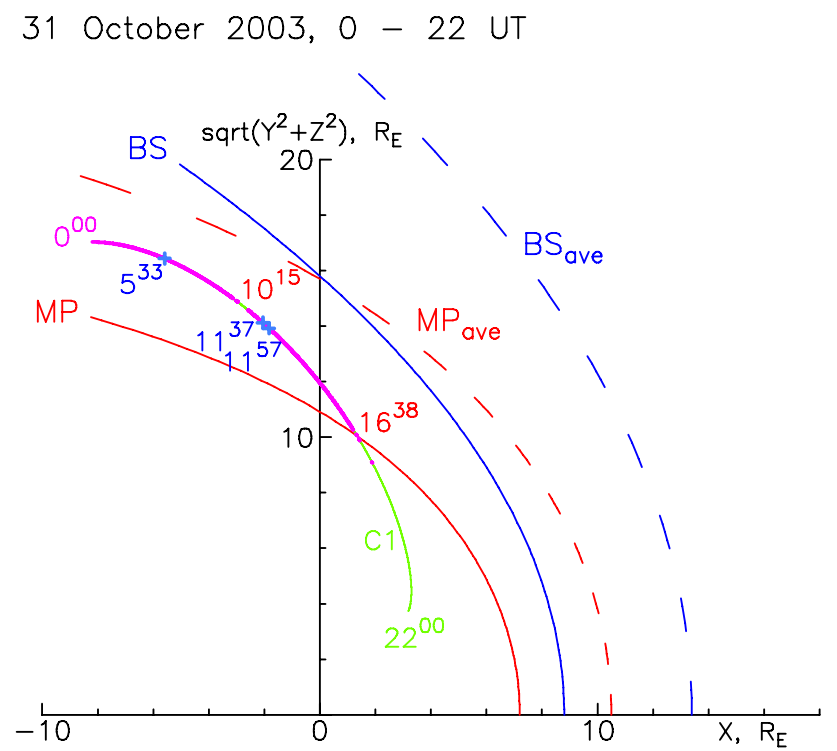

Fig. 6. 31 October 2003. Cluster 1 inbound trajectory in cylindrical coordinates. Lilac sections of orbit are in magnetosheath, green line is in magnetosphere. Times of bow shock crossings are marked by blue crosses. BS2 model (Cairns et al., 1995; Farris and Russell, 1994) bow shock is shown by blue lines and MP1 model (Shue et al., 1998) magnetopause by red lines: dashed curves for average solar wind parameters, solid curves calculated with parameters around 11:57 UT when the spacecraft crossed the bow shock.

Between 09:40 and 10:50 UT, multiple magnetopause crossings were simultaneously observed (with negligible time delay) by all four Cluster satellites, which were separated by $300-400 \mathrm{~km}$. Inbound crossings are marked by dashed vertical lines; outbound crossings are shown by solid lines. The four magnetospheric intervals are indicated by plasma velocity and density dropping to very low values (as measured by CIS/HIA aboard Cluster 1) and by magnetic field increasing to a value between $50-60 \mathrm{nT}$ exhibiting only minor variations. The second time interval between 11:35 and 12:00 UT presents multiple bow shock crossings as observed by Cluster 1 and Cluster 4. For this time interval, plasma parameters are provided by the CIS/CODIF instrument. Inbound crossings are marked by dotted vertical lines; outbound crossings are shown by solid lines. The different transit times through the shock indicate that Cluster 4, which was about $100 \mathrm{~km}$ further upstream in the direction of the bow shock normal, spent a longer time in the interplanetary field compared to Cluster 1.

The OMNI dataset provides all parameters from about 11:15 UT, after the recovery of the ACE plasma detector. Figure 8 shows model predictions for the location of the bow shock and the magnetopause for angular directions of the Cluster 1 position between 11:15 and 19:00 UT. As seen in the top panel, neither of the models provides a good prediction for the bow shock location at 11:37 and 11:57 UT (indicated by vertical lines). The difference in distance between model calculations and observations is larger than $3 R_{\mathrm{E}}$ in the $\mathrm{SZA}=100^{\circ}$ angular direction (corresponding to $\sim 2.5 R_{\mathrm{E}}$ in the direction of the bow shock normal), even for the BS1 model (Farris et al., 1991; Farris and Russell, 1994) which provides the location closest to the orbit. In panel 2 of Fig. 8 , the calculated magnetopause locations are presented. Dashed line at 16:38 UT indicates the inbound magnetopause crossing which was followed by three short excursions (3$5 \mathrm{~min}$ ) back to the magnetosheath. The three-dimensional MP2 model (Lin et al., 2010) provides better predictions for the observed magnetopause locations compared to the other two models which predict the magnetopause farther upstream than observed. The spacecraft was relatively close to the cusp indentation (at an angle of $20-25^{\circ}$ ) at that time. Model MP2 also predicts the short excursion of the spacecraft to the magnetosphere around 15:55 UT and the multiple magnetopause crossings observed after 16:38 UT until 17:43 UT.

Dmitriev et al. (2005) investigated all magnetopause crossings at geosynchronous orbit during 29-31 October which could be identified from magnetic field measurements of the GOES satellites or from ion and electron spectra detected by four different LANL (Los Alamos National Laboratory) satellites. Several multiple magnetopause crossings around local noon were found to be accompanied by global oscillations of the geomagnetic field with period of 4-6 min. The amplitude of these magnetopause oscillations was estimated at about $0.3-0.6 R_{\mathrm{E}}$ around the nose. Also, a dawn-dusk asymmetry of the magnetopause was revealed when magnetopause crossings observed simultaneously on the morning and afternoon side were compared with estimated magnetopause locations using the models of Shue et al. (1998), Kuznetsov and Suvorova (1998), and Chao et al. (2002). For the time of the largest disturbances, no measured solar wind dynamic pressure data were available. Dmitriev et al. (2005) estimated the lower level of dynamic pressure from magnetosheath intervals, i.e. when the satellite was located in the magnetosheath; the actual pressure was supposed to be higher than the model prediction. On the other hand, the upper level of the pressure was obtained from magnetosphere intervals.

In this study, the upper limit of solar wind dynamic pressure was estimated for the time intervals spent by Cluster 1 in the magnetosphere as it was done by Dmitriev et al. (2005). The actual pressure was supposed to be lower than the value applied in model MP1 (Shue et al., 1998) when providing the observed magnetopause location: $P_{\mathrm{d}}<4.7 \mathrm{nPa}$ between 09:52 and 10:02 UT, while $P_{\mathrm{d}}<4.3 \mathrm{nPa}$ between 10:15 and 10:40 UT. In a similar way, the lower limit of dynamic pressure was estimated for the time intervals when Cluster 1 was upstream of the bow shock, and the actual pressure was supposed to be higher than the value used in model BS2 (Cairns et al., 1995; Farris and Russell, 1994) when providing the observed bow shock location: $P_{\mathrm{d}}>95 \mathrm{nPa}$ between 05:33 and 

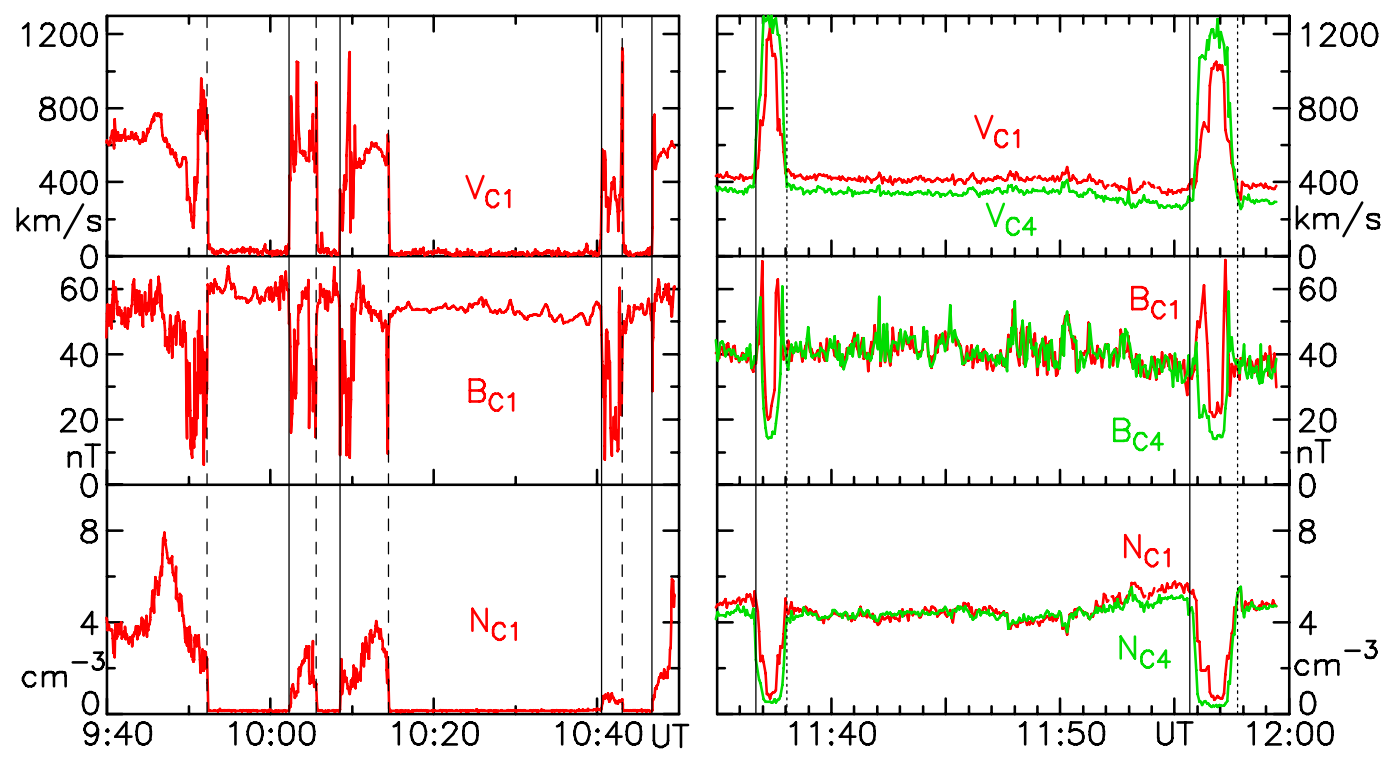

Fig. 7. 31 October 2003, 09:40-10:50 UT and 11:35-12:00 UT. Solar wind velocity $V$, magnetic field $B$, and proton number density $N$ measurements: Cluster 1 in red and Cluster 4 in green. Observed inbound magnetopause crossings are marked by dashed lines; inbound bow shock crossings are marked by dotted lines. Solid lines mark outbound crossings for both discontinuities.

05:43 UT, while $P_{\mathrm{d}}>90 \mathrm{nPa}$ around 11:37 and 11:57 UT. The lower levels of dynamic pressure estimated from the bow shock crossings are in acceptable agreement with the results of Dmitriev et al. (2005), who estimated $P_{\mathrm{d}}>80$ $90 \mathrm{nPa}$ for the time interval 05:33-05:43 UT and $P_{\mathrm{d}}>60$ $70 \mathrm{nPa}$ around 11:30-12:00 UT from magnetopause crossings observed by LANL geosynchronous satellites when using the Shue et al. (1998) model (cf. Fig. 10 in their paper). However, solar wind dynamic pressure measured by ACE between 11:30 and 12:00 UT was much smaller $(20-25 \mathrm{nPa})$ than estimated above and by Dmitriev et al. (2005).

Lopez et al. (2007) reconstructed solar wind plasma data in the vicinity of the Earth using densities from the Geotail plasma wave experiment and velocities from ACE (data for alpha particles were available) for 29 and 30 October. Based on these estimated interplanetary parameters, the LyonFedder-Mobarry (LFM) three-dimensional MHD code (cf. Lyon et al., 2004) was used to simulate magnetic field along the GOES 10 and GOES 12 trajectory. Magnetosheath intervals provided by the LFM model were compared with the predictions of three empirical models (Petrinec and Russell, 1993; Roelof and Sibeck, 1993; Shue et al., 1998). According to verification statistics for each model against GOES observations, the LFM model provided the best forecasting results for magnetopause crossings. Also, in agreement with the results of Dmitriev et al. (2005), a significant local time asymmetry was found in the magnetopause position.

\section{Discussion}

Observed bow shock and magnetopause locations were presented in Sect. 3 for three events when these boundaries were unusually close to the Earth due to large interplanetary disturbances. The observations were compared with the predictions of models which use different methods for describing the location of the discontinuity. MP1 (Shue et al., 1998) is a two-dimensional model which provides simple equations for the relations between interplanetary solar wind conditions (dynamic pressure and IMF $B_{\mathrm{Z}}$ ) and magnetopause parameters (standoff distance and flaring angle). MP2 (Lin et al., 2010) is a three-dimensional magnetopause model which includes the effect of the dipole tilt angle and the magnetic pressure of the interplanetary field (in addition to dynamic pressure and IMF $B_{\mathrm{Z}}$ ). MP3 is a new two-dimensional model (developed from Verigin et al., 2009) which takes into account the IMF component transverse to the solar wind flow and the dynamic pressure.

BS1 uses a simple equation of an ellipsoid of revolution for describing the shape of the bow shock. The eccentricity was determined for average conditions by Farris et al. (1991), but actual solar wind measurements are used in this work for calculating the standoff distance (Farris and Russell, 1994) based on the magnetopause standoff distance provided by MP2 (Lin et al., 2010). BS2 provides a twodimensional bow shock shape depending on the actual solar wind dynamic pressure (Cairns et al., 1995), and the standoff distance is calculated from the formula given by Farris and Russell (1994) using the magnetopause standoff distance determined by MP1 (Shue et al., 1998). BS3 is a 


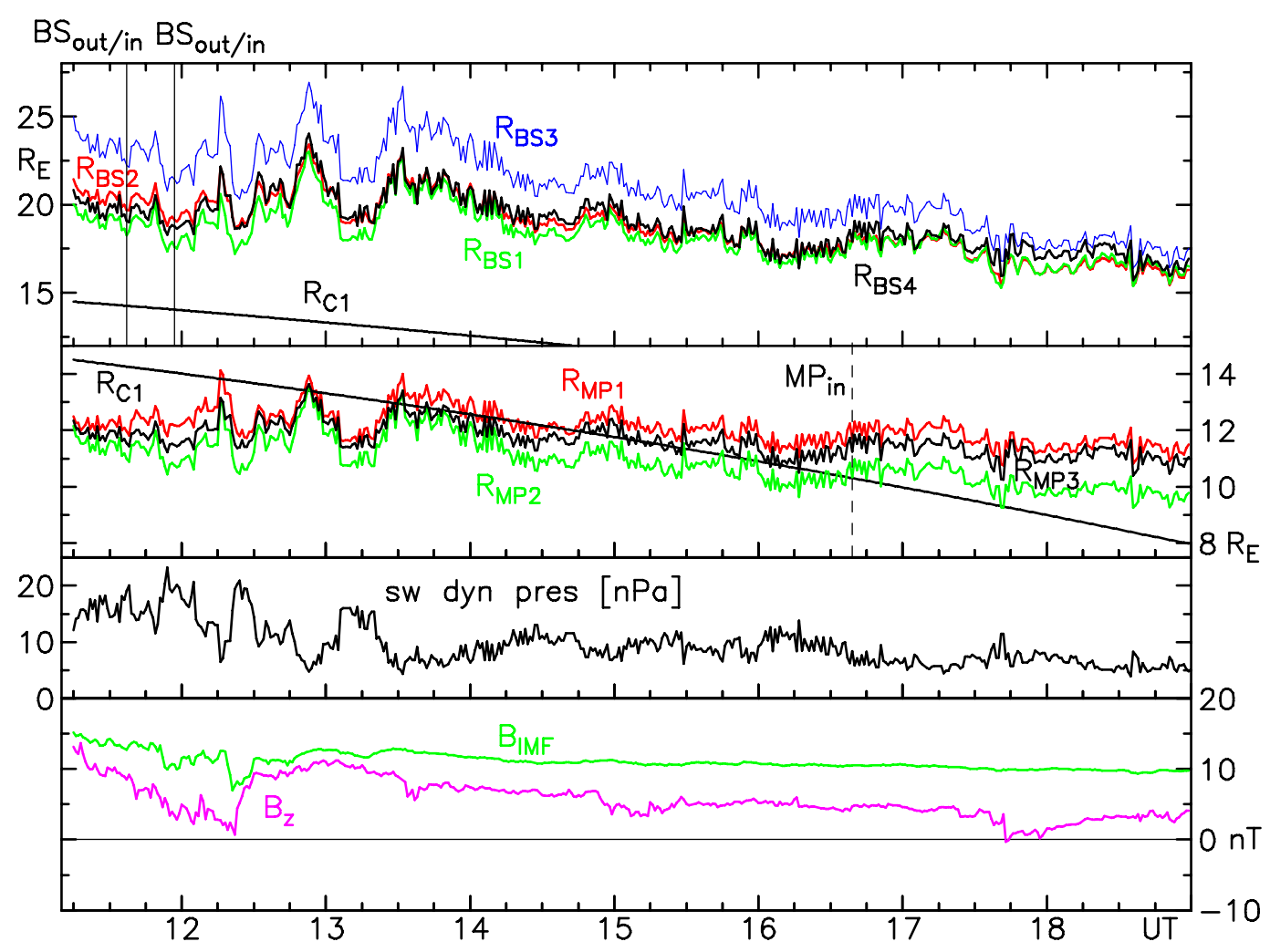

Fig. 8. 31 October 2003. Top panel: model bow shock locations for angular directions of Cluster 1 position: BS1 in green (Farris et al., 1991; Farris and Russell, 1994), BS2 in red (Cairns et al., 1995; Farris and Russell, 1994), BS3 in blue (Jerab et al., 2005), and BS4 in black (Verigin et al., 2001a,b, 2003); spacecraft trajectory $R_{\mathrm{C} 1}$ is the black line. Observed bow shock crossings (out- and inbound around 11:37 and 11:57 UT) are marked by vertical lines. Panel 2: model magnetopause locations for Cluster 1 angular directions: MP1 in red (Shue et al., 1998), MP2 in green (Lin et al., 2010), and MP3 in black (new model); spacecraft trajectory $R_{\mathrm{C} 1}$ is the black line; dashed line is magnetopause crossing. Panel 3: solar wind dynamic pressure. Bottom panel: IMF total value (green) and $B_{\mathrm{Z}}$ (lilac). Interplanetary data from ACE measurements.

three-dimensional empirical model in the GSE system using the actual solar wind dynamic pressure, Alfvénic Mach number, and the total value of the IMF (Jerab et al., 2005). BS4 is a three-dimensional semi-empiric model combined with MHD solution using the GIPM reference frame (Verigin et al., 2001a,b, 2003). It uses measured solar wind velocity, density, magnetic field, and Mach numbers. The magnetopause standoff distance and curvature radius are taken from MP3 (new model). While BS1 is an arbitrary model, BS2 and earlier versions of BS3 were analyzed in comparative studies of Merka et al. (2003, 2005a,b). BS4 was investigated by Merka et al. (2005a,b).

Figure 9a and b show the magnetopause and bow shock profiles provided by the three magnetopause and four bow shock models applying the solar wind parameters measured at 13:00 UT and 16:30 UT on 17 January 2005 when extreme solar wind conditions were observed. The vertical axis pointing upwards represents the + Y-direction, while the vertical axis pointing downwards represents the $-Z$-direction for the three-dimensional model profiles. The 3-D bow shock models BS3 (Jerab et al., 2005) and BS4 (Verigin et al., 2001a,b, 2003) are shown in GSE coordinate system while the 3-D magnetopause model MP2 (Lin et al., 2010) is presented in GSM coordinates (the rotation angle from GSE to GSM was $2.2^{\circ}$ at $13: 00 \mathrm{UT}$ and $10^{\circ}$ at $16: 30 \mathrm{UT}$ ). As seen in Figs. 1 and 4, the IMF was very large, and dynamic pressure was higher than average around 13:00 UT: $B=40 \mathrm{nT}$, $B_{\mathrm{z}} \approx 0 \mathrm{nT}$ and $P_{\mathrm{d}}=15 \mathrm{nPa}$. Around 16:30 UT, however, the IMF value was average, but dynamic pressure was extremely large: $B=5 \mathrm{nT}, B_{\mathrm{Z}}=+4 \mathrm{nT}$, and $P_{\mathrm{d}}=53 \mathrm{nPa}$.

As seen in Fig. 9a, large $B$ (with large $B_{\mathrm{y}}$, but $B_{\mathrm{z}}=0$ ) and moderately large $P_{\mathrm{d}}$ provide significant differences between the predictions of the magnetopause models. The magnetopause provided by MP3 (new model) is more than $1 R_{\mathrm{E}}$ closer to the Earth at the nose compared to the other two models due to the large $B_{\mathrm{y}}$ component. The difference between MP1 (Shue et al., 1998) and MP3 (new model) slightly increases towards the flanks. MP2 (Lin et al., 2010) is located between MP1 and MP3, the cusp indentation is at $49^{\circ}$ solar zenith angle in the $X /-Z_{\mathrm{GSM}}$ section. The differences between the bow shock locations provided by models BS1 (Farris et al., 1991; Farris and Russell, 1994), BS2 (Cairns et 

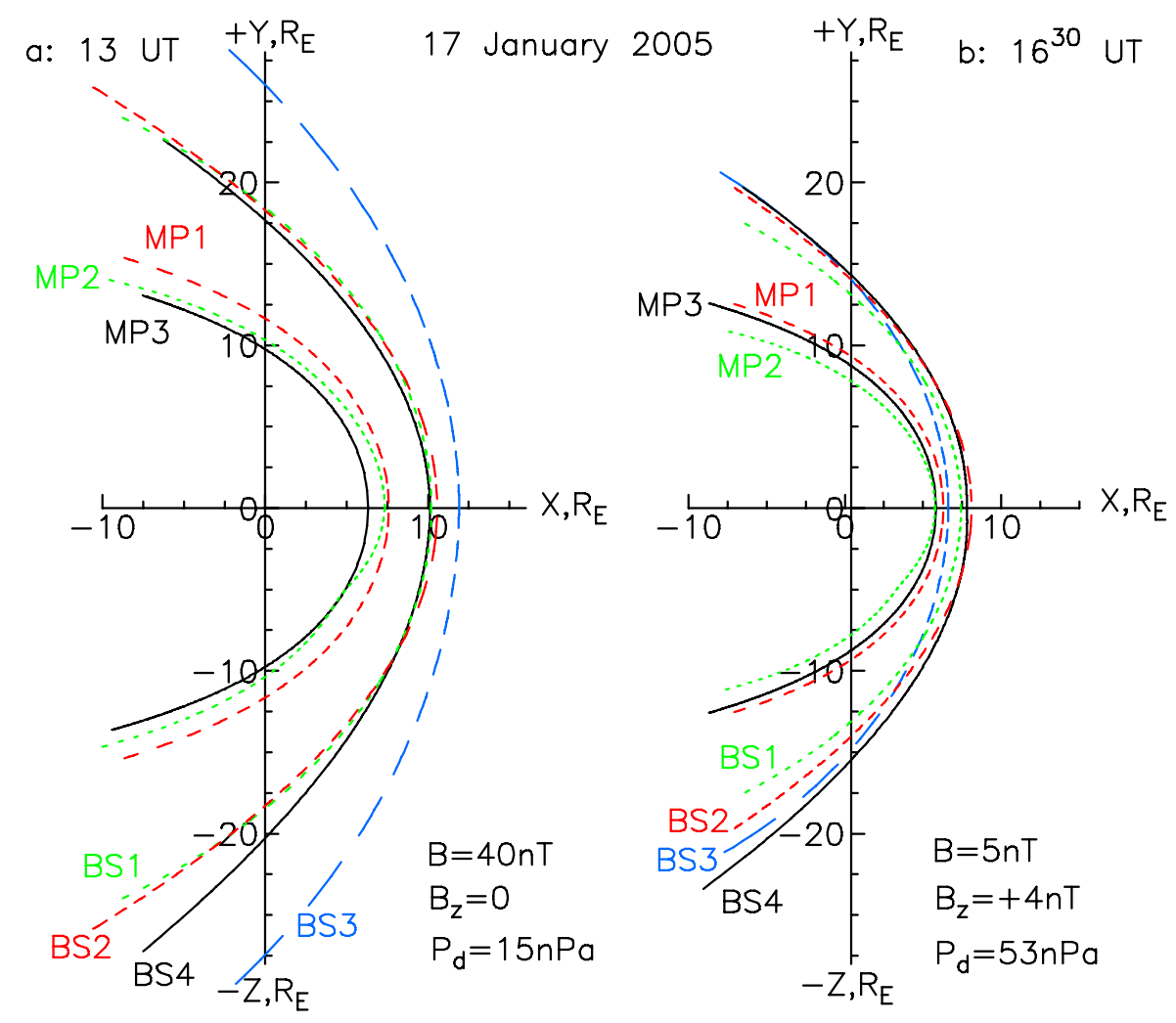

Fig. 9. Magnetopause and bow shock profiles provided by the three magnetopause and four bow shock models when using the solar wind parameters measured (a) at 13:00 UT and (b) at 16:30 UT on 17 January 2005: MP1 (Shue et al., 1998) in dashed red, MP2 (Lin et al., 2010) in dotted green, and MP3 (new model) in continuous black line; BS1 (Farris et al., 1991; Farris and Russell, 1994) in dotted green, BS2 (Cairns et al., 1995; Farris and Russell, 1994) in dashed red, BS3 (Jerab et al., 2005) in dashed blue, and BS4 (Verigin et al., 2001a,b, 2003) in continuous black line. Vertical axis pointing upwards: $+Y_{\mathrm{GSE}}$ direction; vertical axis pointing downwards: $-Z_{\mathrm{GSE}}$ direction $(\mathrm{MP} 2$ model is in GSM coordinates).

al., 1995; Farris and Russell, 1994), and BS4 (Verigin et al., 2001a,b, 2003) are within $0.5 R_{\mathrm{E}}$ in the $X /+Y$ section. In the $X /-Z$ section, the deviation of the 3-D BS4 model from the 2-D models increases towards the flank. The 3-D BS3 model (Jerab et al., 2005), which is more influenced by the value of the IMF (see parameter $D$ in Eq. 5), predicts the bow shock location significantly farther upstream compared to the other models.

Figure $9 \mathrm{~b}$ shows that the deviations between the different magnetopause and bow shock models are smaller when the dynamic pressure is extremely large and the IMF is average, as measured around 16:30 UT. In this case, the 3-D MP2 model (Lin et al., 2010) provides the closest location to the Earth, the cusp indentation (at $54^{\circ} \mathrm{SZA}$ ) almost disappears due to the large compression. The magnetopause profile provided by MP1 (Shue et al., 1998) is farther away from the Earth by about $0.4 R_{\mathrm{E}}$ at the nose, and the deviation increases towards the flanks. The MP3 profile (new model) is between MP1 and MP2 at all solar zenith angles. The 2-D bow shock model BS2 (Cairns et al., 1995; Farris and Russell, 1994) and the 3-D model BS4 (Verigin et al., 2001a,b, 2003) are very similar in the $X /+Y$ section; the deviation of the other two bow shock models is more significant. BS1 (Farris et al., 1991; Farris and Russell, 1994) is located closer to the Earth than BS2 as the standoff distance of BS1 is based on the magnetopause standoff distance of MP2, which is smaller than that of MP1 used for BS2. According to Fig. 9a and 9b, the shapes of BS1 and BS2 are similar in the investigated SZA ranges, in spite of the fact that the flaring angle of BS2 is influenced by the dynamic pressure while the eccentricity of BS1 is constant. The nose distance of BS3 (Jerab et al., 2005) is the smallest, while its flaring angle is the largest.

For average solar wind conditions, as measured around 07:00 UT on 17 January 2005, the differences between the predictions of the different magnetopause and bow shock models were found to be smaller. The only exception is the 3-D BS3 model (Jerab et al., 2005), which deviates significantly from the other three models; namely the flaring angle of the bow shock is larger as seen also in Fig. 9a and b. Therefore, the distance to the nose is smaller, while the distance at the terminator is larger. 
In all three events presented in Sect. 3, MP1 (Shue et al., 1998) provided the outermost location for the magnetopause compared to the other two models, as illustrated also by Fig. 9a and b. On 17 January 2005 when dynamic pressure was very large but $B_{\mathrm{Z}}$ values were small, the predictions of MP1 seem to be the best for the location of GOES 12, supposing that the satellite was in the magnetosheath only between 15:05 and 17:41 UT. Magnetopause locations provided by the other two models are also within $0.2 R_{\mathrm{E}}$ of the satellite's orbit for about $1 \mathrm{~h}$ before 15:05 UT and after 17:41 UT when these models predict magnetosheath intervals. In lack of plasma data, it is difficult to tell the exact times of magnetopause crossings as IMF $B_{\mathrm{Z}}$ was positive during these time intervals. For 20 November 2003, however, when the IMF $B_{\mathrm{Z}}$ component was negative for more than $12 \mathrm{~h}$, it is obvious that the satellites entered the magnetosheath when they observed negative $B_{\mathrm{Z}}$ in the nose regions. For this event, magnetosheath intervals predicted by models MP2 (Lin et al., 2010) and MP3 (new model) agree better with observations compared to model MP1 (Shue et al., 1998) for both GOES 10 and GOES 12. It seems that the saturation effect is not properly taken into account in Eq. (1a) for large negative $B_{Z}$ values. Chao et al. (2002) modified the model of Shue et al. (1998) by providing different formulas for the standoff distance and for the flaring angle in different ranges of $B_{\mathrm{Z}}$. Also, the coefficients are different in their formulas in different ranges of $R_{\mathrm{MP}_{0}}$. Yang et al. (2002) found that the forecasting capability of the model of Chao et al. (2002) is better for the prediction of geosynchronous magnetopause crossings compared to the model of Shue et al. (1998) and also to the model of Petrinec and Russell (1996).

The magnetopause crossing observed by Cluster 1 at 21:41 UT on 17 January 2005 is much better predicted by models MP3 (new model) and MP2 (Lin et al., 2010) compared to model MP1 (Shue et al., 1998). On 31 October 2003, the magnetopause crossing at 16:38 UT is closest to the predictions of the 3-D MP2 model, which also indicates the multiple crossings observed earlier and later. In both cases, the spacecraft was relatively close to the cusp (at an angle of $22^{\circ}-24^{\circ}$ ) when the magnetopause crossings were observed. Dynamic pressure and magnetic field were larger than average values, but not extreme.

From the presented bow shock crossings observed by Cluster 1, only the one at 19:51 UT on 17 January 2005 could be well predicted by BS1, which is an arbitrary combination of the models of Farris et al. (1991) and Farris and Russell (1994) using the magnetopause standoff distance from the model of Lin et al. (2010). Neither of the models predicted bow shock crossings around 10:25 and 10:42 UT on 17 January 2005 , shown in Fig. $3\left(\mathrm{SZA} \approx 60^{\circ}\right.$ ), and around 11:37 and 11:57 UT on 31 October 2003, shown in Fig. 7 (SZA $\approx 100^{\circ}$ ). In the first case, the calculated bow shock locations were closer to the Earth than observed by about $2 R_{\mathrm{E}}$; in the second case they were farther upstream by about $2.5 R_{\mathrm{E}}$ in the bow shock normal direction. On both days, interplanetary parameters changed significantly when the discussed bow shock crossings were observed. Accordingly, all four models predicted displacements of the bow shock towards the Cluster 1 orbit, but the provided locations were not close enough to the trajectory of the spacecraft.

Under disturbed solar wind conditions, statistical models based on the values of interplanetary parameters in an equilibrium state cannot always give good predictions for the sudden displacement of the discontinuities, as they may be affected also by indirect effects. Global MHD simulations showed (Samsonov et al., 2007) that a moderately strong interplanetary fast shock, interacting with the Earth's magnetosphere, will be reflected from the ionosphere. The passage of the inbound transmitted shock causes the bow shock and the magnetopause to move inward, while the passage of the sunward-propagating reflected shock causes the boundaries to move out, as confirmed by Pallocchia et al. (2010) based on observations aboard Double Star TC1 and Cluster 3. Safrankova et al. (2007) suggested that the combination of the inward and outward motions of the bow shock, caused by the transition of an interplanetary shock, results in an indentation of the bow shock surface which flows along the bow shock together with the interplanetary shock. The motion of the indentation can be recorded as two bow shock crossings separated by $1-5 \mathrm{~min}$. Safrankova et al. (2007) interpreted out- and inbound bow shock crossing pairs observed by Interball 1 and Geotail as the result of the passage of an interplanetary shock through the magnetosheath.

Jelinek et al. (2010) found several cases in THEMIS observations when the whole magnetosheath was swept along the probes in a few minutes due to the large displacement of the bow shock and the magnetopause as a result of their interaction with solar wind discontinuities. In one case, upstream observations did not reveal any cause of the observed magnetopause displacement, because solar wind parameters were nearly stable except for the IMF $B_{\mathrm{x}}$ and $B_{\mathrm{y}}$ components which changed proportions. The change in the direction of the field was different as observed by ACE and Wind. Jelinek et al. (2010) supposed that a tangential discontinuity evolved between the location of ACE and the Earth. They suggested that the IMF orientation (not simply the variations in $B_{\mathrm{Z}}$ ) may control the magnetopause and bow shock positions.

In order to illustrate the capabilities of a global threedimensional magnetohydrodynamic model, simulations were performed at NASA's Community Coordinated Modeling Center (http://ccmc.gsfc.nasa.gov/) using the SWMF BATSRUS code. The same upstream solar wind and magnetic field parameters were used as for the empirical models; they were measured by Wind for 17 January 2005 and by ACE for 31 October 2003. The Earth's magnetic field was approximated by a dipole with the actual axis orientation. In Fig. 3, thin black lines present the velocity, magnetic field and proton number density profiles determined by the 3-D MHD model for the Cluster 1 trajectory on 17 January 2005. As 
indicated by the decrease of velocity, and increase of magnetic field and proton number density, the CCMC model run predicts an excursion to the magnetosheath around 10:30 UT which is slightly shorter than observed by Cluster 1 . The direction of the solar wind was out of the ecliptic plane by $8-13^{\circ}$ for more than $1 \mathrm{~h}$ around the time of the bow shock crossings, and sudden changes occurred in all interplanetary parameters. In such a case, the 3-D MHD model can be expected to provide better prediction for the location of the bow shock. However, the MHD model cannot resolve the thickness of the discontinuity.

As seen in the second time interval of Fig. 3, the global MHD model predicts sudden changes in all parameters between 19:00 and 19:30 UT, which can be interpreted as two in- and outbound bow shock crossings. As seen in Fig. 4, the simple models also predict two out- and inbound displacements of the bow shock, caused by the variations of the interplanetary parameters. The MHD model provides a decrease in velocity and a sudden increase in magnetic field around 19:51 UT, corresponding to the observed time of the bow shock crossing. Around 21:40 UT, magnetic field increases, while velocity and density decrease according to the 3-D MHD model, in good agreement with the observed time of the magnetopause crossing. However, the MHD model cannot resolve the thickness of the discontinuity; the magnetopause transition was faster according to the Cluster $1 \mathrm{ob}-$ servations compared to the model prediction.

The results of a similar 3-D MHD model run at CCMC for 31 October 2003 do not indicate that the bow shock was close to the trajectory of Cluster 1 around 11:37 or 11:57 UT. Around that time, ACE observed a significant increase in solar wind dynamic pressure and a decrease in the IMF magnitude and change in direction. It can be supposed that this structure evolved while propagating to the Earth at a speed of $\sim 1000 \mathrm{~km} \mathrm{~s}^{-1}$, and it raised indentations in the bow shock when passing through the magnetosheath to the location of the Cluster spacecraft at SZA $\approx 100^{\circ}$.

\section{Summary}

Three events were discussed from the declining phase of the last solar cycle when the magnetopause and/or the bow shock were observed unusually close to the Earth due to major interplanetary disturbances. The observed extreme locations of the discontinuities are compared with the predictions of three magnetopause (MP1: Shue et al., 1998; MP2: Lin et al., 2010; MP3: new model based on Verigin et al., 2009) and four bow shock models (BS1: Farris et al., 1991, combined with Farris and Russell, 1994; BS2: Cairns et al., 1995, combined with Farris and Russell, 1994; BS3: Jerab et al., 2005; BS4: Verigin et al., 2001a,b, 2003) which describe them in considerably different ways using statistical methods based on observations, except for BS4, which is a 3-D semi-empiric model combined with MHD solution. Three of the bow shock models are using the magnetopause standoff distance determined by a magnetopause model; BS1 is based on MP2, BS2 on MP1, and BS4 on MP3, as discussed in Sect. 2.2. BS4 also applies the curvature radius of the magnetopause at the nose.

The observed magnetopause crossings could be predicted by one (or more) of the three empirical models with a reasonable accuracy. Under different conditions, however, different models provided the best prediction.

1. On 20 November 2003 when the IMF was extremely large having a large negative $B_{\mathrm{Z}}$ component, the geosynchronous magnetopause crossings observed by the GOES satellites were best predicted by the new model, while the model of Shue et al. (1998) provided the poorest prediction.

2. On 17 January 2005 when solar wind dynamic pressure was extremely large, the predictions of the model of Shue et al. (1998) agreed best with the location of the magnetopause as observed by GOES 12 . In this case, however, the exact time of the magnetopause crossings could not be determined as IMF $B_{\mathrm{Z}}$ was not always negative during the investigated interval and plasma data were not available.

3. The magnetopause crossings observed by Cluster 1 were best predicted by the three-dimensional model of Lin et al. (2010), probably due to the location of the spacecraft being relatively close to the cusp (at an angle of $22^{\circ}-24^{\circ}$ ) on 31 October 2003 and on 17 January 2005. The new model also provided a very good prediction for the latter event.

Bow shock crossings observed by the Cluster spacecraft could not be predicted by either of the empirical models, except for the event at 19:51 UT on 17 January 2005 when the arbitrary model BS1 (Farris et al., 1991; Farris and Russell, 1994) provided the closest prediction. The insufficiency of the statistical models can be explained by the large disturbances in the interplanetary field. Magnetic field discontinuities may evolve while travelling to the Earth from the spacecraft monitoring the solar wind. Also, they can raise new discontinuities when interacting with the terrestrial magnetosphere. Predicting these secondary effects is beyond the capability of the empirical models which use interplanetary parameters measured farther upstream of the bow shock.

A global three-dimensional MHD model of NASA's CCMC predicted the short magnetosheath excursion observed by the Cluster spacecraft on 17 January 2005, proving that this event was caused by the variations of the interplanetary parameters as measured by the Wind spacecraft. According to the results of the 3-D MHD model, the bow shock was not close to the trajectory of Cluster 1 when it observed the two short solar wind intervals on 31 October 2003, indicating that this event was probably caused by secondary effects raised by the evolution of the measured interplanetary disturbances. 
The new magnetopause model introduced in this work has to be carefully tested in the future by studying more cases when the IMF has a large component transverse to the SunEarth direction.

Acknowledgements. This work was supported by FP7 of the European Commission in the framework of project SOTERIA: SolarTerrestrial Investigations and Archives (http://soteria-space.eu/). The authors are thankful to NASA's Community Coordinated Modeling Center for providing 3-D MHD model runs for the selected events. The CDAWeb service of NASA's Goddard Space Flight Center is also acknowledged for providing magnetic field data measured by the GOES satellites and for preparing the OMNI dataset containing interplanetary parameters. Partial support from P-22 and OFN-15 programs by RAS.

Guest Editor M. Taylor thanks Y. Bogdanova and one anonymous referee for their help in evaluating this paper.

\section{References}

Balogh, A., Carr, C. M., Acuña, M. H., Dunlop, M. W., Beek, T. J., Brown, P., Fornacon, K.-H., Georgescu, E., Glassmeier, K.H., Harris, J., Musmann, G., Oddy, T., and Schwingenschuh, K.: The Cluster Magnetic Field Investigation: overview of in-flight performance and initial results, Ann. Geophys., 19, 1207-1217, doi:10.5194/angeo-19-1207-2001, 2001.

Bieber, J. W. and Stone, E. C.: Energetic electron bursts in the magnetopause electron layer and in interplanetary space, in: Proceedings of Magnetospheric Boundary Layers Conference, ESA SP148, 131-135, 1979.

Boardsen, S. A., Eastman, T. E., Sotirelis, T., and Green, J. L.: An empirical model of the high-latitude magnetopause, J. Geophys. Res. 105, 23193-23219, 2000.

Cairns, I. H. and Lyon, J. G.: MHD simulations of the Earth's bow shock at low Mach numbers: Standoff distances, J. Geophys. Res. 100, 17173-17180, 1995.

Cairns, I. H., Fairfield, D. H., Anderson, R. R., Carlton, V. E. H., Paularena, K. I., and Lazarus, A. J.: Unusual locations of the Earth's bow shock on September 24-25, 1987: Mach number effects, J. Geophys. Res., 100, 47-62, 1995.

Chao, J. K., Wu, D. J., Lin, C.-H., Yang, Y. H., Wang, X. Y., Kessel, M., Chen, S. H., and Lepping, R. P.: Models for the size and shape of the Earth's magnetopause and bow shock, in: Space Weather Study Using Multipoint Techniques, COSPAR Colloq. Ser., Vol. 12, edited by: Lyu, L.-H., 127-134, Pergamon, Oxford, 2002.

Crooker, N. U., Siscoe, G. L., Mullen, P. R., Russell, C. T., and Smith, E. J.: Magnetic field compression at the dayside magnetopause, J. Geophys. Res., 87, 10407-10412, 1982.

Dmitriev, A. V. and Suvorova, A. V.: Three-dimensional artificial neural network model of the dayside magnetopause, J. Geophys. Res., 105, 18909-18918, 2000.

Dmitriev, A., Chao, J.-K., Thomsen, M., and Suvorova, A.: Geosynchronous magnetopause crossings on 29-31 October 2003, J. Geophys. Res., 110, A08209, doi:10.1029/2004JA010582, 2005.

Fairfield, D. H.: Average and unusual locations of the Earth's magnetopause and bow shock, J. Geophys. Res., 76, 6700-6715, 1971.
Farris, M. H. and Russell, C. T.: Determining the standoff distance of the bow shock: Mach number dependence and use of models, J. Geophys. Res. 99, 17681-17689, 1994.

Farris, M. H., Petrinec, S. M., and Russell, C. T.: The thickness of the magnetosheath : Constraints on the polytropic index, Geophys. Res. Lett. 18, 1821-1824, 1991.

Ferraro, V. C. A.: On the theory of the first phase of a geomagnetic storm: A new illustrative calculation based on idealized (plane not cylindrical) model field distribution, J. Geophys. Res., 57, $15-49,1952$.

Formisano, V.: Orientation and shape of the Earth's bow shock in three-dimensions, Planet. Space Sci., 27, 1151-1161, 1979.

Formisano, V., Hedgecock, P. C., Moreno, G., Sear, J., and Bollea, D.: Observations of Earth's bow shock for low Mach numbers, Planet. Space Sci., 19, 1519-1531, 1971.

Formisano, V., Domingo, V., and Wenzel, K. P.: The threedimensional shape of the magnetopause, Planet. Space Sci., 27, 1137-1149, 1979.

Howe, H. C. and Binsack, J., H.: Explorer 33 and 35 plasma observations of magnetosheath flow, J. Geophys. Res., 77, 3334-3344, 1972.

Jelinek, K., Nemecek, Z., Safrankova, J., Shue, J.-H., Suvorova, A. V., and Sibeck, D. G.: Thin magnetosheath as a consequence of magnetopause deformation: THEMIS observations, J. Geophys. Res., 115, A10203, doi:10.1029/2010JA015345, 2010.

Jerab, M., Nemecek, Z., Safrankova, J., Jelinek, K., and Merka, J.: Improved bow shock model with dependence on the IMF strength, Planet. Space Sci., 53, 85-93, 2005.

Kuznetsov, S. N. and Suvorova, A. V.: An empirical model of the magnetopause for broad ranges of solar wind pressure and $B_{\mathrm{Z}}$ IMF, in: Polar Cap Boundary Phenomena, NATO ASI Ser., edited by: Moe, J., Egeland, A., and Lockwood, M., 51-61, Springer, New York, 1998.

Lin, R. L., Zhang, X. X., Liu, S. Q., Wang, Y. L., and Gong, J. C.: A three-dimensional asymmetric magnetopause model, J. Geophys. Res., 115, A04207, doi:10.1029/2009JA014235, 2010.

Lopez, R. E., Hernandez, S., Wiltberger, M., Huang, C.-L., Kepko, E. L., Spence, H., Goodrich, C. C., and Lyon, J. G.: Predicting magnetopause crossings at geosynchronous orbit during the Halloween storms, Space Weather, 5, S01005, doi:10.1029/2006SW000222, 2007.

Lyon, J. G., Fedder, J. A., and Mobarry, C. M.: The Lyon-FedderMobarry (LFM) global MHD magnetospheric simulation code, J. Atmos. Sol. Terr. Phys., 66, 1333-1350, 2004.

Merka, J. and Szabo, A.: Bow shock's geometry at the magnetospheric flanks, J. Geophys. Res., 109, A12224, doi:10.1029/2004JA010567, 2004.

Merka, J., Szabo, A., Narock, T. W., King, J. H., Paularena, K. I., and Richardson, J. D.: A comparison of IMP 8 observed bow shock positions with model predictions, J. Geophys. Res., 108, 1077, doi:10.1029/2002JA009384, 2003.

Merka, J., Szabo, A., Narock, T. W., Richardson, J. D., and King, J. H.: Three decades of bow shock observations by IMP 8 and model predictions, Planet. Space Sci., 53, 79-84, 2005a.

Merka, J., Szabo, A., Slavin, J. A., and Peredo, M.: Threedimensional position and shape of the bow shock and their variation with upstream Mach numbers and interplanetary magnetic field orientation, J. Geophys. Res., 110, A04202, doi:10.1029/2004JA010944, 2005b. 
Nemecek, Z. and Safrankova, J.: The Earth's bow shock and magnetopause position as a result of the solar wind - magnetosphere interaction, J. Atmos. Terr. Phys., 53, 1049-1054, 1991.

Pallocchia, G., Samsonov, A. A., Bavassano Cattaneo, M. B., Marcucci, M. F., Rème, H., Carr, C. M., and Cao, J. B.: Interplanetary shock transmitted into the Earth's magnetosheath: Cluster and Double Star observations, Ann. Geophys., 28, 1141-1156, doi:10.5194/angeo-28-1141-2010, 2010.

Peredo, M., Slavin, J. A., Mazur, E., and Curtis, S. A.: Threedimensional position and shape of the bow shock and their variation with Alfvenic, sonic and magnetosonic Mach numbers and interplanetary magnetic field orientation, J. Geophys. Res., 100, 7907-7916, 1995.

Petrinec, S. M. and Russell, C. T.: An empirical model of the size and shape of the near-Earth magnetotail, Geophys. Res. Lett., 20, 2695-2698, 1993.

Petrinec, S. M. and Russell, C. T.: Near-Earth magnetotail shape and size as determined from the magnetopause flaring angle, J. Geophys. Res., 101, 137-152, 1996.

Petrinec, S. P., Song, P., and Russell, C. T.: Solar cycle variations in the size and shape of the magnetopause, J. Geophys. Res., 96, 7893-7896, 1991.

Rème, H., Aoustin, C., Bosqued, J. M., Dandouras, I., Lavraud, B., Sauvaud, J. A., Barthe, A., Bouyssou, J., Camus, Th., Coeur-Joly, O., Cros, A., Cuvilo, J., Ducay, F., Garbarowitz, Y., Medale, J. L., Penou, E., Perrier, H., Romefort, D., Rouzaud, J., Vallat, C., Alcaydé, D., Jacquey, C., Mazelle, C., d'Uston, C., Möbius, E., Kistler, L. M., Crocker, K., Granoff, M., Mouikis, C., Popecki, M., Vosbury, M., Klecker, B., Hovestadt, D., Kucharek, H., Kuenneth, E., Paschmann, G., Scholer, M., Sckopke, N., Seidenschwang, E., Carlson, C. W., Curtis, D. W., Ingraham, C., Lin, R. P., McFadden, J. P., Parks, G. K., Phan, T., Formisano, V., Amata, E., Bavassano-Cattaneo, M. B., Baldetti, P., Bruno, R., Chionchio, G., Di Lellis, A., Marcucci, M. F., Pallocchia, G., Korth, A., Daly, P. W., Graeve, B., Rosenbauer, H., Vasyliunas, V., McCarthy, M., Wilber, M., Eliasson, L., Lundin, R., Olsen, S., Shelley, E. G., Fuselier, S., Ghielmetti, A. G., Lennartsson, W., Escoubet, C. P., Balsiger, H., Friedel, R., Cao, J.-B., Kovrazhkin, R. A., Papamastorakis, I., Pellat, R., Scudder, J., and Sonnerup, B.: First multispacecraft ion measurements in and near the Earth's magnetosphere with the identical Cluster ion spectrometry (CIS) experiment, Ann. Geophys., 19, 1303-1354, doi:10.5194/angeo19-1303-2001, 2001.

Roelof, E. C. and Sibeck, D. G.: Magnetopause shape as a bivariate function of interplanetary magnetic field $B_{z}$ and solar wind dynamic pressure, J. Geophys. Res., 98, 21421-21450, 1993.

Safrankova, J., Nemecek, Z., Prech, L., Samsonov, A. A., Koval, A., and Andreeova, K.: Modification of interplanetary shocks near the bow shock and through the magnetosheath, J. Geophys. Res., 112, A08212, doi:10.1029/2007JA012503, 2007.
Samsonov, A. A., Sibeck, D. G., and Imber, J.: MHD simulation for the interaction of an interplanetary shock with the Earth's magnetosphere, J. Geophys. Res., 112, A12220, doi:10.1029/2007JA012627, 2007.

Shue, J.-H., Chao, J. K., Fu, H. C., Russell, C. T., Song, P., Khurana, K. K., and Singer, H. J.: A new functional form to study the solar wind control of the magnetopause size and shape, J. Geophys. Res., 102, 9497-9511, 1997.

Shue, J.-H., Song, P., Russell, C. T., Steinberg, J. T., Chao, J. K., Zastenker, G., Vaisberg, O. L., Kokubun, S., Singer, H. J., Detman, T. R., and Kawano, H.: Magnetopause location under extreme solar wind conditions, J. Geophys. Res., 103, 17691-17700, 1998.

Sibeck, D. G., Lopez, R. E., and Roelof, E. C.: Solar wind control of the magnetopause shape, location, and motion, J. Geophys. Res., 96, 5489-5495, 1991.

Slavin, J. A. and Holzer, R. E.: Solar wind flow about the terrestrial planets. 1. Modeling bow shock position and shape, J. Geophys. Res., 86, 11401-11418, 1981.

Spreiter, J. R., Summers, A. L., and Alksne, A. Y.: Hydromagnetic flow around the magnetosphere, Planet. Space Sci., 14, 223-253, 1966.

Suvorova, A., Dmitriev, A., Chao, J.-K., Thomsen, M., and Yang, Y.-H.: Necessary conditions for geosynchronous magnetopause crossings, J. Geophys. Res., 110, A01206, doi:10.1029/2003JA010079, 2005.

Tátrallyay, M. and Erdős, G.: The evolution of mirror mode fluctuations in the terrestrial magnetosheath, Planet. Space Sci., 50, 593-599, 2002.

Verigin, M. I., Kotova, G. A., Slavin, J., Szabo, A., Kessel, M., Safrankova, J., Nemecek, Z., Gombosi, T. I., Kabin, K., Shugaev, F., and Kalinchenko, A.: Analysis of the 3-D shape of the terrestrial bow shock by Interball/Magion 4 observations, Adv. Space Res., 28, 857-862, 2001a.

Verigin, M., Kotova, G., Szabo, A., Slavin, J., Gombosi, T., Kabin, K., Shugaev, F., and Kalinchenko, A.: Wind observations of the terrestrial bow shock: 3-D shape and motion, Earth Planets Space, 53, 1001-1009, 2001b.

Verigin, M., Slavin, J., Szabo, A., Kotova, G., and Gombosi, T.: Planetary bow shocks: Asymptotic MHD Mach cones, Earth Planets Space, 55, 33-38, 2003.

Verigin, M. I., Kotova, G. A., Bezrukikh, V. V., Zastenker, G. N., and Nikolaeva, N: Analytical model of the near-Earth magnetopause according to the data of the Prognoz and Interball satellite data, Geomagnetism and Aeronomy, 49, 1176-1181, 2009.

Yang, Y.-H., Chao, J. K., Lin, C.-H., Shue, J.-H., Wang, X.-Y., Song, P., Russell, C. T., Lepping, R. P., and Lazarus, A. J.: Comparison of three magnetopause prediction models under extreme solar wind conditions, J. Geophys. Res., 107, 1008, doi:10.1029/2001JA000079, 2002. 